Fully discrete scheme for Bean's critical-state model with temperature effects in superconductivity

Winckler, Malte; Yousept, Irwin

This text is provided by DuEPublico, the central repository of the University Duisburg-Essen.

This version of the e-publication may differ from a potential published print or online version.

URN: urn:nbn:de:hbz:464-20180910-112727-6

Link: https://duepublico.uni-duisburg-essen.de:443/servlets/DocumentServlet?id=46818

Source: Deutsche Forschungsgemeinschaft (DFG), Priority Programme 1962: SPP 1962;

Preprint Number SPP1962-065 https://spp1962.wias-berlin.de/preprints.php 


\title{
FULLY DISCRETE SCHEME FOR BEAN'S CRITICAL-STATE MODEL WITH TEMPERATURE EFFECTS IN SUPERCONDUCTIVITY*
}

\author{
M. WINCKLER ${ }^{\dagger}$ AND I. YOUSEPT ${ }^{\dagger}$
}

\begin{abstract}
This paper examines the fully discrete analysis of a hyperbolic Maxwell-type variational inequality with temperature effects arising from Bean's critical-state model in type-II (hightemperature) superconductivity. Here, temperature dependence is included in the critical current due to its main importance for the realization of superconducting effects, as confirmed through physical measurements. We propose a fully discrete scheme based on the implicit Euler in time and a mixed FEM in space consisting of Nédélec's edge elements for the electric field and piecewise constant elements for the magnetic induction. Under a regularity assumption on the initial data and a specific setting of the initial approximation, we prove that the fully discrete scheme admits a unique solution satisfying the discrete Gauss law, which turns out to be the key point for our analysis. Hereafter, stability estimates for the zero-oder and first-order terms of the proposed approximation are investigated. Our main result is the strong convergence of the fully discrete scheme without any further regularity assumption. In particular, the convergence result yields the existence of a unique solution for the variational inequality obeying the physical Gauss law. The final part of the paper is devoted to the a priori error analysis under an additional Lipschitz assumption on the critical current and the temperature distribution as well as a higher regularity assumption on the continuous solution. We close this paper by presenting some 3D numerical results, which in particular confirm the physical Meissner-Ochsenfeld effect in superconductivity.
\end{abstract}

Key words. Maxwell variational inequality, Bean's critical-state model with temperature effects, Gauss law, superconductivity, fully discrete scheme, convergence analysis, error estimates.

AMS subject classifications. 35Q61, 35L87, 78M10

1. Introduction. The physical phenomenon of superconductivity is characterized by zero electrical resistance and repulsion of magnetic fields (Meissner-Ochsenfeld effect) under the condition that the temperature is below some critical level. It was first discovered in 1911 by H. Kamerlingh-Onnes and has gained tremendous theoretical and practical attentions ever since. Nowadays, modern magnetic levitation trains, distributed superconducting magnetic energy storage (D-SMES), magnetic resonance imaging (MRI) and magnetic confinement fusion cannot be realized without the use of superconductors, just to mention a few key technologies. A critical-state model describing the magnetization process of penetration and exit of magnetic flux in type-II (high-temperature) superconductors was proposed by Bean [4,5]. More precisely, his model describes a nonlinear and non-smooth constitutive relation between the (total) current density and the electric field as follows:

(B1) The current density strength $|\mathbf{J}|$ cannot exceed the critical current $j_{c}$;

(B2) if $|\mathbf{J}|$ is strictly less than $j_{c}$, then the electric field $\mathbf{E}$ vanishes;

(B3) the electric field $\mathbf{E}$ is parallel to $\mathbf{J}$.

We underline that the (unknown) superconductive region is determined by points $(x, t) \in \Omega \times(0, T)$, for which the strict inequality $|\mathbf{J}(x, t)|<j_{c}$ is satisfied. Thus, in this region, there is no electrical resistance so that the electric field must vanish.

\footnotetext{
* Submitted to the editors 28.05.2018

Funding: This work was supported by the German Research Foundation Priority Program DFG SPP 1962 "Non-smooth and Complementarity-based Distributed Parameter Systems: Simulation and Hierarchical Optimization", Project YO 159/2-1.

†University of Duisburg-Essen, Fakultät für Mathematik, Thea-Leymann-Str. 9, D-45127 Essen, Germany, malte.winckler@uni-due.de, irwin.yousept@uni-due.de.
} 
The Bean critical-state model (B1)-(B3) governed by the eddy current equations with magnetic field dependence $j_{c}=j_{c}(\mathbf{H})$ leads to a parabolic quasi-variational inequality (QVI) of obstacle type. Prigozhin [23, 24] was the first, who introduced and analyzed this formulation. Barrett and Prigozhin [2] analyzed it in a scalar twodimensional (2D) setting and its dual formulation. The finite element analysis for the associated parabolic variational inequality in a 2D setting was investigated in [10] (see also [11] for a similar 2D model using an E-J-formulation). Furthermore, the numerical analysis for the 3D setting was investigated in [9]. Recent results on the numerical analysis for the parabolic QVI in a 2D setting were obtained in [3]. All the previously mentioned contributions were devoted to the numerical analysis for the eddy current case. In the full Maxwell case (cf. [13]), the Bean's critical state model (B1)-(B3) with $j_{c}=j_{c}(x)$ leads to a mixed hyperbolic variational inequality of the second kind [26]. The numerical analysis for this variational inequality is still in its earlier state. We are only aware of the recent work [26] for the analysis of the semi-discrete spatial Galerkin approximations.

This paper is devoted to the fully discrete analysis of the Bean critical-state model (B1)-(B3) governed by the full Maxwell equations with temperature effects. Let us underline that in all previously mentioned contributions, temperature dependence was neglected. However, by the nature of superconductivity, temperature effects play a major role, since superconducting effects strongly depend on the temperature itself and can only be reached, if the temperature is underneath some critical level. We refer to $[1,8]$ concerning experimental measurements showing the strong temperature dependence in the critical current $j_{c}=j_{c}(x, \theta(x, t))$ and its physical properties. Let us now formulate the variational inequality we focused on in this paper:

$$
\left\{\begin{array}{l}
\int_{\Omega} \epsilon \partial_{t} \mathbf{E}(t) \cdot(\mathbf{v}-\mathbf{E}(t))+\mu^{-1} \partial_{t} \mathbf{B}(t) \cdot(\mathbf{w}-\mathbf{B}(t)) d x \\
\quad+\int_{\Omega} \mu^{-1} \mathbf{c u r l} \mathbf{E}(t) \cdot \mathbf{w}-\mu^{-1} \mathbf{B}(t) \cdot \mathbf{c u r l} \mathbf{v} d x \\
\quad+\varphi(\theta(t), \mathbf{v})-\varphi(\theta(t), \mathbf{E}(t)) \geq \int_{\Omega} \mathbf{f}(t) \cdot(\mathbf{v}-\mathbf{E}(t)) d x \\
\quad \text { for a.e. } t \in(0, T) \text { and every }(\mathbf{v}, \mathbf{w}) \in \mathbf{H}_{0}(\mathbf{c u r l}) \times \mathbf{L}^{2}(\Omega), \\
\quad(\mathbf{E}(0), \mathbf{B}(0))=\left(\mathbf{E}_{0}, \mathbf{B}_{0}\right)
\end{array}\right.
$$

with a nonsmooth $L^{1}$-type functional

$$
\varphi: L^{\infty}(\Omega) \times \mathbf{L}^{1}(\Omega) \rightarrow \mathbb{R}, \quad(y, \mathbf{v}) \mapsto \int_{\Omega} j_{c}(x, y(x))|\mathbf{v}(x)| d x
$$

In this setting, $\Omega \subset \mathbb{R}^{3}$ is a bounded, polyhedral and simply connected domain with a connected Lipschitz-boundary. Furthermore, $\mathbf{E}: \Omega \times(0, T) \rightarrow \mathbb{R}^{3}$ denotes the electric field, $\mathbf{B}: \Omega \times(0, T) \rightarrow \mathbb{R}^{3}$ the magnetic induction, $\mathbf{f}: \Omega \times(0, T) \rightarrow \mathbb{R}^{3}$ the applied current source and $\theta: \Omega \times(0, T) \rightarrow \mathbb{R}^{3}$ the temperature distribution. Note that in (VI) and all what follows, we use the abbreviation $\mathbf{E}(t)=\mathbf{E}(\cdot, t)$ (the same notation is also used for other quantities). The precise mathematical assumptions for the data involved in (VI) will be given in Section 2.

In $\left(\mathrm{VI}_{N, h}\right)$, we propose a fully discrete scheme for (VI) based on the implicit Euler in time and a mixed FEM in space consisting of Nédélec's edge elements [22] for $\mathbf{E}$ and piecewise constant elements for $\mathbf{B}$. Furthermore, we consider finite element approximations for the initial data $\left(\mathbf{E}_{0}, \mathbf{B}_{0}\right)$ by solving an elliptic curl-curl 
variational inequality (3.1). Under this specific setting and relying on an appropriate regularity assumption on $\left(\mathbf{E}_{0}, \mathbf{B}_{0}\right)$, we are able to prove that $\left(\mathrm{VI}_{N, h}\right)$ admits a unique solution satisfying the discrete Gauss law (Theorem 3.3). Hereafter, we derive stability estimates for the zero-order and first-order terms of the fully discrete solution (Lemmas 3.5 and 3.6). Our main result (Theorem 3.8) is the strong convergence of $\left(\mathrm{VI}_{N}, h\right)$ towards $(\mathrm{VI})$, which in particular implies the existence of a unique solution for (VI) satisfying the physical Gauss law

$$
\operatorname{div} \mathbf{B}=\operatorname{div}(\epsilon \mathbf{E})=0 .
$$

We note that Gauss' law play an important role for Maxwell's equations, both from the mathematical and physical point of views. We refer the recent work by Ciarlet et al. [6] on a unified edge element method for electromagnetic problems with strong convergence in the Gauss law. In our case, the Gauss law turns out to be vital for proving the strong convergence of $\left(\mathrm{VI}_{N, h}\right)$. This seems to be the first indication of its essential impact in the numerical analysis of Maxwell-type variational inequalities. The final part of the paper is devoted to the a priori error analysis for the proposed fully discrete scheme $\left(\mathrm{VI}_{N, h}\right)$. Under an additional Lipschitz assumption on $j_{c}$ and $\theta$ as well as a higher regularity assumption on the solution of (VI), we derive a priori estimates for the $\mathbf{L}^{2}(\Omega)$-error between the fully discrete solution and the continuous one (Theorem 4.4). Last but not least, we refer the reader to some existing works $[7,16,17,20]$ concerning fully discrete approximations for time-dependent Maxwell's equations.

2. Preliminaries. For a given Banach space $X$, we denote its norm by $\|\cdot\|_{X}$ and the duality pairing with the corresponding dual space $X^{*}$ by $\langle\cdot, \cdot\rangle$. If $X$ is a Hilbert space, then $(\cdot, \cdot)_{X}$ stands for its scalar product and $\|\cdot\|_{X}$ for the induced norm. In the case of $X=\mathbb{R}^{n}$, we renounce the subscript in the (Euclidean) norm and write $|\cdot|$. The Euclidean scalar product is denoted by a dot. Unless otherwise stated, we identify the dual space $X^{*}$ with the Hilbert space $X$ itself. The embedding between two Banach spaces $X, Y$ is denoted by $X \hookrightarrow Y$. Now, we introduce the central Hilbert space throughout this paper:

$$
\mathbf{H}(\operatorname{curl}):=\left\{\mathbf{v} \in \mathbf{L}^{2}(\Omega): \operatorname{curl} \mathbf{v} \in \mathbf{L}^{2}(\Omega)\right\},
$$

where curl is understood in the distributional sense. Also, note that we use bold letters for vector-valued functions and the respective spaces. As usual, $\mathbf{C}_{0}^{\infty}(\Omega)$ denotes the space of all infinitely differentiable functions with compact support in $\Omega$. The space $\mathbf{H}_{0}(\mathbf{c u r l})$ stands for the closure of $\mathbf{C}_{0}^{\infty}(\Omega)$ with respect to the $\mathbf{H}(\mathbf{c u r l})$-norm. Furthermore, the spaces of divergence-free vector functions are

$$
\begin{aligned}
& \mathbf{H}(\operatorname{div}=0):=\left\{\mathbf{v} \in \mathbf{L}^{2}(\Omega):(\mathbf{v}, \nabla \phi)_{\mathbf{L}^{2}(\Omega)}=0 \quad \forall \phi \in H_{0}^{1}(\Omega)\right\}, \\
& \mathbf{H}_{0}(\operatorname{div}=0):=\left\{\mathbf{v} \in \mathbf{L}^{2}(\Omega):(\mathbf{v}, \nabla \phi)_{\mathbf{L}^{2}(\Omega)}=0 \quad \forall \phi \in H^{1}(\Omega)\right\},
\end{aligned}
$$

which are endowed with the $\mathbf{L}^{2}(\Omega)$-norm. Another important Hilbert space used throughout this paper is

$$
\mathbf{X}^{(\epsilon)}(\Omega):=\mathbf{H}_{0}(\mathbf{c u r l}) \cap \epsilon^{-1} \mathbf{H}(\operatorname{div}=0) .
$$

Material parameters will occur on the problem statement and thus, for a given positive function $\alpha \in L^{\infty}(\Omega)$, we denote by $\mathbf{L}_{\alpha}^{2}(\Omega)$ the weighted $\mathbf{L}^{2}(\Omega)$-space with the weighted scalar product $(\alpha \cdot, \cdot)_{\mathbf{L}^{2}(\Omega)}$. Moreover, we denote by $C>0$ a generic constant, that can change during an estimation. Let us close this section by presenting all the mathematical assumptions for (VI). 
Assumption 2.1 (Regularity assumptions on the material parameters).

(A1) The material parameters $\epsilon, \mu \in L^{\infty}(\Omega)$ are strictly positive, i.e., there exist positive constants $\underline{\epsilon}, \bar{\epsilon}, \underline{\mu}, \bar{\mu} \in \mathbb{R}_{>0}$ such that

$$
\underline{\epsilon} \leq \epsilon(x) \leq \bar{\epsilon} \quad \text { and } \quad \underline{\mu} \leq \mu(x) \leq \bar{\mu} \text { for a.e. } x \in \Omega .
$$

(A2) The critical current $j_{c}: \Omega \times \mathbb{R} \rightarrow \mathbb{R}$ is a Carathéodory function. To be more precise, for every fixed $y \in \mathbb{R}$, the mapping $j_{c}(\cdot, y): \Omega \rightarrow \mathbb{R}$ is Lebesguemeasurable and, for almost every fixed $x \in \Omega$, the mapping $j_{c}(x, \cdot): \mathbb{R} \rightarrow \mathbb{R}$ is continuous.

(A3) The critical current $j_{c}: \Omega \times \mathbb{R} \rightarrow \mathbb{R}$ is with respect to the second variable (temperature) locally bounded; i.e., for every $M>0$, there exists a constant $C(M)>0$ such that

$$
0 \leq j_{c}(x, y) \leq C(M)
$$

holds for a.e. $x \in \Omega$ and every $y \in[-M, M]$.

Let us remark that the continuity property (A2) and the local boundedness property (A3) for the temperature dependence in the critical current are justified by experimental measurements reported in $[1,8]$.

Assumption 2.2 (Regularity assumptions on the given data).

(A4) Suppose that $\mathbf{f} \in \mathcal{C}^{0,1}([0, T], \mathbf{H}(\operatorname{div}=0))$ and $\theta \in \mathcal{C}\left([0, T], L^{\infty}(\Omega)\right)$.

(A5) The initial data $\left(\mathbf{E}_{0}, \mathbf{B}_{0}\right) \in \mathbf{X}^{(\epsilon)}(\Omega) \times \mathbf{H}_{0}(\operatorname{div}=0)$ satisfies the compability system

$$
\left\{\begin{array}{l}
\int_{\Omega} \epsilon \mathbf{E}_{0} \cdot\left(\mathbf{v}-\mathbf{E}_{0}\right)+\mu^{-1} \mathbf{B}_{0} \cdot\left(\mathbf{w}-\mathbf{B}_{0}\right) d x \\
\quad+\int_{\Omega} \mu^{-1} \mathbf{c u r l} \mathbf{E}_{0} \cdot \mathbf{w}-\mu^{-1} \mathbf{B}_{0} \cdot \mathbf{c u r l} \mathbf{v} d x \\
\quad+\varphi(\theta(0), \mathbf{v})-\varphi\left(\theta(0), \mathbf{E}_{0}\right) \geq \int_{\Omega} \mathbf{f}(0) \cdot\left(\mathbf{v}-\mathbf{E}_{0}\right) d x \\
\quad \text { for all }(\mathbf{v}, \mathbf{w}) \in \mathbf{H}_{0}(\mathbf{c u r l}) \times \mathbf{L}^{2}(\Omega) .
\end{array}\right.
$$

3. Fully discrete scheme. As pointed out in the introduction, we focus on the implicit Euler scheme for the time discretization in (VI). To this aim, let us fix $N \in \mathbb{N}$ and define an equidistant partition of $[0, T]$ in the following way:

$$
\tau:=\frac{T}{N}, \quad 0=t_{0}<t_{1}<\cdots<t_{N}=T \quad \text { with } \quad t_{n}:=n \tau
$$

for all $n \in\{0, \ldots, N\}$. Furthermore, we define

$$
\mathbf{f}^{n}:=\mathbf{f}\left(t_{n}\right) \in \mathbf{H}(\operatorname{div}=0), \quad \varphi^{n}(\mathbf{v}):=\int_{\Omega} j_{c}\left(x, \theta\left(x, t_{n}\right)\right)|\mathbf{v}(x)| d x \quad \forall n \in\{0, \ldots, N\} .
$$

We choose a family of quasi-uniform triangulations $\left\{\mathcal{T}_{h}\right\}_{h>0}$, i.e.,

$$
\bar{\Omega}=\bigcup_{T \in \mathcal{T}_{h}} T \quad \forall h>0
$$

and, for $h_{T}$ denoting the diameter of $T$ and $\rho_{T}$ denoting the diameter of the largest ball contained in $T$, there exist constants $\rho>0$ and $\nu>0$ such that

$$
\frac{h_{T}}{\rho_{T}} \leq \rho \quad \text { and } \quad \frac{h}{h_{T}} \leq \nu \quad \forall T \in \mathcal{T}_{h} .
$$


The subscript $h$ denotes the maximum of $h_{T}$ for $T \in \mathcal{T}_{h}$. The finite element space of Nédélec's first family of edge elements is defined by

$$
\mathbf{V}_{h}:=\left\{\mathbf{v}_{h} \in \mathbf{H}_{0}(\mathbf{c u r l}):\left.\mathbf{v}_{h}\right|_{T}=\mathbf{a}_{T}+\mathbf{b}_{T} \times x \text { with } \mathbf{a}_{T}, \mathbf{b}_{T} \in \mathbb{R}^{3}, \forall T \in \mathcal{T}_{h}\right\}
$$

and the finite element space of piecewise constant functions is denoted by

$$
\mathbf{W}_{h}:=\left\{\mathbf{w}_{h} \in \mathbf{L}^{2}(\Omega):\left.\mathbf{w}_{h}\right|_{T}=\mathbf{a}_{T} \text { with } \mathbf{a}_{T} \in \mathbb{R}^{3}, \forall T \in \mathcal{T}_{h}\right\},
$$

which satisfy curl $\mathbf{V}_{h} \subset \mathbf{W}_{h}$. In addition to these spaces, we introduce the space of continuous piecewise linear elements with vanishing traces by

$$
\Theta_{h}:=\left\{\phi_{h} \in H_{0}^{1}(\Omega):\left.\phi_{h}\right|_{T}=\mathbf{a}_{T} \cdot x+b_{T} \text { with } \mathbf{a}_{T} \in \mathbb{R}^{3}, b_{T} \in \mathbb{R} \quad \forall T \in \mathcal{T}_{h}\right\} .
$$

By means of the above space, the subspace $\mathbf{X}_{h}^{(\epsilon)} \subset \mathbf{V}_{h}$ is defined by

$$
\mathbf{X}_{h}^{(\epsilon)}:=\left\{\mathbf{v}_{h} \in \mathbf{V}_{h}:\left(\epsilon \mathbf{v}_{h}, \nabla \psi_{h}\right)_{\mathbf{L}^{2}(\Omega)^{2}(\Omega)}=0 \quad \forall \psi_{h} \in \Theta_{h}\right\},
$$

i.e., it consists of all discrete $\epsilon$-divergence-free edge element functions.

Having introduced all the required finite element spaces, we now propose the following fully discrete scheme to (VI):

\section{$\left(\mathrm{VI}_{N, h}\right)$}

$$
\left\{\begin{array}{l}
\int_{\Omega} \epsilon \delta \mathbf{E}_{h}^{n} \cdot\left(\mathbf{v}_{h}-\mathbf{E}_{h}^{n}\right)+\mu^{-1} \delta \mathbf{B}_{h}^{n} \cdot\left(\mathbf{w}_{h}-\mathbf{B}_{h}^{n}\right) d x \\
\quad+\int_{\Omega} \mu^{-1} \operatorname{curl} \mathbf{E}_{h}^{n} \cdot \mathbf{w}_{h}-\mu^{-1} \mathbf{B}_{h}^{n} \cdot \mathbf{c u r l} \mathbf{v}_{h} d x \\
\quad+\varphi^{n}\left(\mathbf{v}_{h}\right)-\varphi^{n}\left(\mathbf{E}_{h}^{n}\right) \geq \int_{\Omega} \mathbf{f}^{n} \cdot\left(\mathbf{v}_{h}-\mathbf{E}_{h}^{n}\right) d x \\
\quad \text { for every }\left(\mathbf{v}_{h}, \mathbf{w}_{h}\right) \in \mathbf{V}_{h} \times \mathbf{W}_{h} \text { and } n \in\{1, \ldots, N\} \\
\quad\left(\mathbf{E}_{h}^{0}, \mathbf{B}_{h}^{0}\right)=\left(\mathbf{E}_{0 h}, \mathbf{B}_{0 h}\right),
\end{array}\right.
$$

where

$$
\delta \mathbf{E}_{h}^{n}:=\frac{\mathbf{E}_{h}^{n}-\mathbf{E}_{h}^{n-1}}{\tau} \quad \text { and } \quad \delta \mathbf{B}_{h}^{n}:=\frac{\mathbf{B}_{h}^{n}-\mathbf{B}_{h}^{n-1}}{\tau} \quad \forall n \in\{1, \ldots, N\},
$$

as well as

$$
\delta \mathbf{E}_{h}^{0}:=\mathbf{E}_{0 h} \quad \text { and } \quad \delta \mathbf{B}_{h}^{0}:=\mathbf{B}_{0 h} .
$$

Moreover, $\left(\mathbf{E}_{0 h}, \mathbf{B}_{0 h}\right) \in \mathbf{V}_{h} \times \mathbf{W}_{h}$ denotes the finite element approximation of $\left(\mathbf{E}_{0}, \mathbf{B}_{0}\right)$, which is specified as follows:

Assumption 3.1. Let $\left(\mathbf{E}_{0 h}, \mathbf{B}_{0 h}\right) \in \mathbf{V}_{h} \times \mathbf{W}_{h}$ satisfy the discrete compability system

$$
\left\{\begin{array}{l}
\int_{\Omega} \epsilon \mathbf{E}_{0 h} \cdot\left(\mathbf{v}_{h}-\mathbf{E}_{0 h}\right)+\mu^{-1} \mathbf{B}_{0 h} \cdot\left(\mathbf{w}_{h}-\mathbf{B}_{0 h}\right) d x \\
\quad+\int_{\Omega} \mu^{-1} \mathbf{c u r l} \mathbf{E}_{0 h} \cdot \mathbf{w}_{h}-\mu^{-1} \mathbf{B}_{0 h} \cdot \mathbf{c u r l} \mathbf{v}_{h} d x \\
\quad+\varphi\left(\theta(0), \mathbf{v}_{h}\right)-\varphi\left(\theta(0), \mathbf{E}_{0 h}\right) \geq \int_{\Omega} \mathbf{f}(0) \cdot\left(\mathbf{v}_{h}-\mathbf{E}_{0 h}\right) d x \\
\quad \text { for all }\left(\mathbf{v}_{h}, \mathbf{w}_{h}\right) \in \mathbf{V}_{h} \times \mathbf{W}_{h} .
\end{array}\right.
$$


Assumption 3.1 is indeed reasonable, since (3.1) admits a unique $\left(\mathbf{E}_{0 h}, \mathbf{B}_{0 h}\right) \in \mathbf{V}_{h} \times$ $\mathbf{W}_{h}$. This follows from the classical theory of variational inequalities [18], as (3.1) can be equivalently formulated as an elliptic curl-curl variational inequality (cf. the proof of Theorem 3.3). Thanks to Assumption 3.1, it makes sense to set $\delta \mathbf{E}_{h}^{0}=\mathbf{E}_{0 h}$ and $\delta \mathbf{B}_{h}^{0}=\mathbf{B}_{0 h}$.

Lemma 3.2. Under Assumptions 2.1, 2.2, and 3.1, the discrete approximation $\left(\mathbf{E}_{0 h}, \mathbf{B}_{0 h}\right) \in \mathbf{V}_{h} \times \mathbf{W}_{h}$ satisfies $\mathbf{E}_{0 h} \in \mathbf{X}_{h}^{(\epsilon)}$ and $\mathbf{B}_{0 h} \in \mathbf{H}_{0}(\operatorname{div}=0)$ for all $h>0$ and

$$
\lim _{h \rightarrow 0}\left\|\mathbf{E}_{0 h}-\mathbf{E}_{0}\right\|_{\mathbf{L}_{\epsilon}^{2}(\Omega)}=\lim _{h \rightarrow 0}\left\|\mathbf{B}_{0 h}-\mathbf{B}_{0}\right\|_{\mathbf{L}_{1 / \mu}^{2}(\Omega)}=0 .
$$

Proof. A crucial point in the following proof is the existence of the following continuous linear operators (see [26, Lemma 4.3] and [21, Theorem 2.45]):

$$
\boldsymbol{\Pi}_{h}: \mathbf{H}_{0}(\operatorname{div}=0) \rightarrow \operatorname{curl} \mathbf{V}_{h} \subset \mathbf{W}_{h} \quad \text { and } \quad \boldsymbol{\Phi}_{h}: \mathbf{H}_{0}(\operatorname{curl}) \rightarrow \mathbf{V}_{h}
$$

with the convergence properties

$$
\begin{aligned}
& \lim _{h \rightarrow 0}\left\|\boldsymbol{\Pi}_{h} \mathbf{w}-\mathbf{w}\right\|_{\mathbf{L}_{1 / \mu}^{2}(\Omega)}=0 \quad \forall \mathbf{w} \in \mathbf{H}_{0}(\operatorname{div}=0), \\
& \lim _{h \rightarrow 0}\left\|\mathbf{\Phi}_{h} \mathbf{v}-\mathbf{v}\right\|_{\mathbf{L}_{\epsilon}^{2}(\Omega)}=0 \quad \forall \mathbf{v} \in \mathbf{H}_{0}(\mathbf{c u r l}) .
\end{aligned}
$$

By testing (2.1) with $(\mathbf{v}, \mathbf{w})=\left(\mathbf{E}_{0 h}, \mathbf{B}_{0 h}\right)$ and (3.1) with $\left(\mathbf{v}_{h}, \mathbf{w}_{h}\right)=\left(\boldsymbol{\Phi}_{h} \mathbf{E}_{0}, \boldsymbol{\Pi}_{h} \mathbf{B}_{0}\right)$, strong convergence follows readily from the above properties after summarizing the resulting inequalities and some rearrangements. Furthermore, inserting $\left(\mathbf{v}_{h}, \mathbf{w}_{h}\right)=$ $\left(2 \mathbf{E}_{0 h}, \mathbf{B}_{0 h}\right)$ and $\left(\mathbf{v}_{h}, \mathbf{w}_{h}\right)=\left(0, \mathbf{B}_{0 h}\right)$ into (3.1), it follows that

$$
\begin{aligned}
\int_{\Omega} \epsilon \mathbf{E}_{0 h} \cdot \mathbf{E}_{0 h} d x-\int_{\Omega} \mu^{-1} \mathbf{B}_{0 h} \cdot \operatorname{curl} \mathbf{E}_{0 h} d x+ & \varphi\left(\theta(0), \mathbf{E}_{0 h}\right) \\
& =\int_{\Omega} \mathbf{f}(0) \cdot \mathbf{E}_{0 h} d x \quad \forall h>0 .
\end{aligned}
$$

Analogously, inserting $(\mathbf{v}, \mathbf{w})=\left(2 \mathbf{E}_{0}, \mathbf{B}_{0}\right)$ and $(\mathbf{v}, \mathbf{w})=\left(0, \mathbf{B}_{0}\right)$ into $(2.1)$ yields

$$
\int_{\Omega} \epsilon \mathbf{E}_{0} \cdot \mathbf{E}_{0} d x-\int_{\Omega} \mu^{-1} \mathbf{B}_{0} \cdot \operatorname{curl} \mathbf{E}_{0} d x+\varphi\left(\theta(0), \mathbf{E}_{0}\right)=\int_{\Omega} \mathbf{f}(0) \cdot \mathbf{E}_{0} d x .
$$

We recall that $\nabla \psi_{h} \in \mathbf{V}_{h} \subset \mathbf{H}_{0}$ (curl) holds for every $\psi_{h} \in \Theta_{h}$ according to the well-known discrete de Rham diagram [21, p. 150]. Hence, we deduce from (2.1) and (3.1)-(3.3) as well as $\operatorname{curl} \nabla \equiv 0$ and $\mathbf{f}(0) \in \mathbf{H}(\operatorname{div}=0)$ that

$$
\int_{\Omega} \epsilon \mathbf{E}_{0 h} \cdot \nabla \psi_{h} d x=\int_{\Omega} \epsilon \mathbf{E}_{0} \cdot \nabla \psi_{h} d x \quad \forall \psi_{h} \in \Theta_{h} \quad \forall h>0 .
$$

Thus, since $\epsilon \mathbf{E}_{0} \in \mathbf{H}(\operatorname{div}=0)$, it follows that $\mathbf{E}_{0 h} \in \mathbf{X}_{h}^{(\epsilon)}$. Finally, inserting $\mathbf{v}_{h}=\mathbf{E}_{0 h}$ in (3.1), we obtain that

$$
\int_{\Omega} \mu^{-1}\left(\mathbf{B}_{0 h}+\operatorname{curl} \mathbf{E}_{0 h}\right) \cdot\left(\mathbf{w}_{h}-\mathbf{B}_{0 h}\right) d x=0 \quad \forall \mathbf{w}_{h} \in \mathbf{W}_{h} .
$$

Since curl $\mathbf{V}_{h} \subset \mathbf{W}_{h}$, we may set $\mathbf{w}_{h}:=2 \mathbf{B}_{0 h}+\operatorname{curl} \mathbf{E}_{0 h}$ in (3.5), which implies

$$
\mathbf{B}_{0 h}=-\operatorname{curl} \mathbf{E}_{0 h} \quad \text { a.e. in } \Omega \text {. }
$$

241 Thus, $\mathbf{B}_{0 h} \in \mathbf{H}_{0}(\operatorname{div}=0)$ follows. 
The following theorem proves the well-posedness of $\left(\mathrm{VI}_{N, h}\right)$ and gives an important regularity property for the discrete solution.

TheOREm 3.3. Let Assumptions 2.1, 2.2, and 3.1 hold. Then, for every $h>0$ and $N \in \mathbb{N}$, the system of discrete variational inequalities $\left(\mathrm{VI}_{N, h}\right)$ admits a unique solution $\left\{\left(\mathbf{E}_{h}^{n}, \mathbf{B}_{h}^{n}\right)\right\}_{n=1}^{N} \subset \mathbf{V}_{h} \times \mathbf{W}_{h}$. Furthermore, the solution enjoys the regularity property $\left\{\left(\mathbf{E}_{h}^{n}, \mathbf{B}_{h}^{n}\right)\right\}_{n=1}^{N} \subset \mathbf{X}_{h}^{(\epsilon)} \times \mathbf{H}_{0}(\operatorname{div}=0)$.

Proof. Let $n \in\{1, \ldots, N\}$ with $N \in \mathbb{N}$ and $h>0$. Furthermore, assume that $\left(\mathbf{E}_{h}^{n-1}, \mathbf{B}_{h}^{n-1}\right)$ is already known. Using the same arguments as in (3.6), we may decouple the variational inequality into two parts by testing $\left(\mathrm{VI}_{N, h}\right)$ with $\mathbf{v}_{h}=\mathbf{E}_{h}^{n}$ to obtain that

$$
\delta \mathbf{B}_{h}^{n}=-\operatorname{curl} \mathbf{E}_{h}^{n} \quad \text { a.e. in } \Omega .
$$

By definition, (3.7) yields the following explicit formula for $\mathbf{B}_{h}^{n}$ :

$$
\mathbf{B}_{h}^{n}=\mathbf{B}_{h}^{n-1}-\tau \operatorname{curl} \mathbf{E}_{h}^{n} \quad \text { a.e. in } \Omega .
$$

With this formula and Lemma 3.2 at hand, we obtain that $\mathbf{B}_{h}^{n} \in \mathbf{W}_{h} \cap \mathbf{H}_{0}(\operatorname{div}=0)$ by inductive reasoning. Next, we insert $\mathbf{w}_{h}=\mathbf{B}_{h}^{n}$ in $\left(\mathrm{VI}_{N, h}\right)$ and employ (3.8) to obtain the variational inequality

$$
\begin{aligned}
& \int_{\Omega} \epsilon \delta \mathbf{E}_{h}^{n} \cdot\left(\mathbf{v}_{h}-\mathbf{E}_{h}^{n}\right) d x+\int_{\Omega} \tau \mu^{-1} \operatorname{curl} \mathbf{E}_{h}^{n} \cdot \operatorname{curl}\left(\mathbf{v}_{h}-\mathbf{E}_{h}^{n}\right) d x+\varphi^{n}\left(\mathbf{v}_{h}\right) \\
& -\varphi^{n}\left(\mathbf{E}_{h}^{n}\right) \geq \int_{\Omega} \mathbf{f}^{n} \cdot\left(\mathbf{v}_{h}-\mathbf{E}_{h}^{n}\right)+\mu^{-1} \mathbf{B}_{h}^{n-1} \cdot \operatorname{curl}\left(\mathbf{v}_{h}-\mathbf{E}_{h}^{n}\right) d x \quad \forall \mathbf{v}_{h} \in \mathbf{V}_{h} .
\end{aligned}
$$

The well-posedness of (3.9) is covered by a classical result in [18], because it is equivalent to an elliptic curl-curl variational inequality of the form

$$
a\left(\mathbf{E}_{h}^{n}, \mathbf{v}_{h}-\mathbf{E}_{h}^{n}\right)+\varphi^{n}\left(\mathbf{v}_{h}\right)-\varphi^{n}\left(\mathbf{E}_{h}^{n}\right) \geq\left\langle\tilde{\mathbf{f}}^{n}, \mathbf{v}_{h}-\mathbf{E}_{h}^{n}\right\rangle \quad \forall \mathbf{v}_{h} \in \mathbf{V}_{h}
$$

with the continuous and coercive bilinear form $a: \mathbf{V}_{h} \times \mathbf{V}_{h} \rightarrow \mathbb{R}$ defined by

$$
a\left(\mathbf{u}_{h}, \mathbf{v}_{h}\right):=\int_{\Omega} \tau^{-1} \epsilon \mathbf{u}_{h} \cdot \mathbf{v}_{h} d x+\int_{\Omega} \tau \mu^{-1} \operatorname{curl} \mathbf{u}_{h} \cdot \operatorname{curl} \mathbf{v}_{h} d x \quad \forall \mathbf{u}_{h}, \mathbf{v}_{h} \in \mathbf{V}_{h}
$$

and the right-hand side $\tilde{\mathbf{f}}^{n} \in \mathbf{H}_{0}(\mathbf{c u r l})^{*}$ by

$$
\left\langle\tilde{\mathbf{f}}^{n}, \mathbf{v}\right\rangle:=\int_{\Omega}\left(\mathbf{f}^{n}+\tau^{-1} \epsilon \mathbf{E}_{h}^{n-1}\right) \cdot \mathbf{v} d x+\int_{\Omega} \mu^{-1} \mathbf{B}_{h}^{n-1} \cdot \operatorname{curl} \mathbf{v} d x \quad \forall \mathbf{v} \in \mathbf{H}_{0}(\mathbf{c u r l}) .
$$

Inserting the solution $\mathbf{E}_{h}^{n}$ of (3.9) into (3.8), we finally obtain a unique solution

$$
\left\{\left(\mathbf{E}_{h}^{n}, \mathbf{B}_{h}^{n}\right)\right\}_{n=1}^{N} \subset \mathbf{V}_{h} \times \mathbf{W}_{h} \cap \mathbf{H}_{0}(\operatorname{div}=0)
$$

of $\left(\mathrm{VI}_{N, h}\right)$. In order to prove the regularity $\mathbf{E}_{h}^{n} \in \mathbf{X}_{h}^{(\epsilon)}$, we test the $n$-th inequality in $\left(\mathrm{VI}_{N, h}\right)$ with $\mathbf{w}_{h}=\mathbf{B}_{h}^{n}$ and the $(n-1)$-th with $\mathbf{w}_{h}=\mathbf{B}_{h}^{n-1}$. Thereafter, we subtract 
the resulting inequalities to conclude that

$$
\begin{aligned}
& \int_{\Omega} \epsilon\left(\delta \mathbf{E}_{h}^{n}-\delta \mathbf{E}_{h}^{n-1}\right) \cdot \mathbf{v}_{h} d x-\int_{\Omega} \mu^{-1}\left(\mathbf{B}_{h}^{n}-\mathbf{B}_{h}^{n-1}\right) \cdot \operatorname{curl} \mathbf{v}_{h} d x \\
& \quad-\int_{\Omega} \epsilon \delta \mathbf{E}_{h}^{n} \cdot \mathbf{E}_{h}^{n} d x+\int_{\Omega} \mu^{-1} \mathbf{B}_{h}^{n} \cdot \mathbf{c u r l} \mathbf{E}_{h}^{n} d x-\varphi^{n}\left(\mathbf{E}_{h}^{n}\right) \\
& \quad+\int_{\Omega} \epsilon \delta \mathbf{E}_{h}^{n-1} \cdot \mathbf{E}_{h}^{n-1} d x-\int_{\Omega} \mu^{-1} \mathbf{B}_{h}^{n-1} \cdot \operatorname{curl} \mathbf{E}_{h}^{n-1} d x+\varphi^{n-1}\left(\mathbf{E}_{h}^{n-1}\right) \\
& \geq \int_{\Omega}\left(\mathbf{f}^{n}-\mathbf{f}^{n-1}\right) \cdot \mathbf{v}_{h} d x-\int_{\Omega} \mathbf{f}^{n} \cdot \mathbf{E}_{h}^{n} d x+\int_{\Omega} \mathbf{f}^{n-1} \cdot \mathbf{E}_{h}^{n-1} d x \quad \forall \mathbf{v}_{h} \in \mathbf{V}_{h} .
\end{aligned}
$$

In the same manner as in (3.2), it is readily seen that

$$
\int_{\Omega} \epsilon \delta \mathbf{E}_{h}^{j} \cdot \mathbf{E}_{h}^{j} d x-\int_{\Omega} \mu^{-1} \mathbf{B}_{h}^{j} \cdot \operatorname{curl} \mathbf{E}_{h}^{j} d x+\varphi^{n}\left(\mathbf{E}_{h}^{j}\right)=\int_{\Omega} \mathbf{f}^{j} \cdot \mathbf{E}_{h}^{j} d x
$$

for $j \in\{n-1, n\}$. By combining (3.11) and (3.12), it follows that

$$
\begin{aligned}
& \int_{\Omega} \epsilon\left(\delta \mathbf{E}_{h}^{n}-\delta \mathbf{E}_{h}^{n-1}\right) \cdot \mathbf{v}_{h} d x-\int_{\Omega} \mu^{-1}\left(\mathbf{B}_{h}^{n}-\mathbf{B}_{h}^{n-1}\right) \cdot \operatorname{curl}_{h} d x \\
&=\int_{\Omega}\left(\mathbf{f}^{n}-\mathbf{f}^{n-1}\right) \cdot \mathbf{v}_{h} d x \quad \forall \mathbf{v}_{h} \in \mathbf{V}_{h} .
\end{aligned}
$$

Therefore, by (A4) and $\operatorname{curl} \nabla \equiv 0$, setting $\mathbf{v}_{h}=\nabla \psi_{h}$ for $\psi_{h} \in \Theta_{h}$ in (3.13) yields

$$
\int_{\Omega} \epsilon\left(\delta \mathbf{E}_{h}^{n}-\delta \mathbf{E}_{h}^{n-1}\right) \cdot \nabla \psi_{h} d x=0 \quad \forall \psi_{h} \in \Theta_{h} .
$$

Now, we see that the above variational equality holds for every $n \in\{1, \ldots, N\}$. Thus, the fact $\mathbf{E}_{0 h} \in \mathbf{X}_{h}^{(\epsilon)}$ (cf. Lemma 3.2) implies that

$$
\left(\epsilon \mathbf{E}_{h}^{n}, \nabla \psi_{h}\right)=0 \quad \forall \psi_{h} \in \Theta_{h} \quad \Rightarrow \quad \mathbf{E}_{h}^{n} \in \mathbf{X}_{h}^{(\epsilon)} \quad \forall n \in\{1, \ldots, N\} .
$$

Remark 3.4. We note that (3.15) is the discrete version of the Gauss law for the electric field, i.e., $\operatorname{div}(\epsilon \mathbf{E})=0$. The discrete Gauss law will be a crucial point for our convergence analysis in Theorem 3.8. Also, the formulas (3.8) and (3.9) will be the foundation for the computation of the numerical solution.

The following Lemmas prove the zero-order and first-order stability estimates for the fully discrete solution to $\left(\mathrm{VI}_{N, h}\right)$ :

Lemma 3.5. Let Assumptions 2.1, 2.2, and 3.1 be satisfied. Then, there exists a constant $C>0$, depending only on $\mathbf{f}, \mathbf{E}_{0}, \mathbf{B}_{0}$ and $T, \epsilon, \mu$, such that for every $N \in \mathbb{N}$ and $h>0$, the solution $\left\{\left(\mathbf{E}_{h}^{n}, \mathbf{B}_{h}^{n}\right)\right\}_{n=1}^{N}$ of $\left(\mathrm{VI}_{N, h}\right)$ fulfills the estimate

$$
\begin{aligned}
& \max _{n \in\{1, \ldots, N\}}\left\|\mathbf{E}_{h}^{n}\right\|_{\mathbf{L}_{\epsilon}^{2}(\Omega)}^{2}+\max _{n \in\{1, \ldots, N\}}\left\|\mathbf{B}_{h}^{n}\right\|_{\mathbf{L}_{1 / \mu}^{2}(\Omega)}^{2} \\
& +\sum_{n=1}^{N}\left\|\mathbf{E}_{h}^{n}-\mathbf{E}_{h}^{n-1}\right\|_{\mathbf{L}_{\epsilon}^{2}(\Omega)}^{2}+\sum_{n=1}^{N}\left\|\mathbf{B}_{h}^{n}-\mathbf{B}_{h}^{n-1}\right\|_{\mathbf{L}_{1 / \mu}^{2}(\Omega)}^{2}+\tau \sum_{n=1}^{N} \varphi^{n}\left(\mathbf{E}_{h}^{n}\right) \leq C .
\end{aligned}
$$


Proof. We start again by testing $\left(\mathrm{VI}_{N, h}\right)$ with $\left(\mathbf{v}_{h}, \mathbf{w}_{h}\right)=\left(2 \mathbf{E}_{h}^{n}, 2 \mathbf{B}_{h}^{n}\right)$ as well as with $\left(\mathbf{v}_{h}, \mathbf{w}_{h}\right)=(0,0)$ to obtain

$$
\int_{\Omega} \epsilon \delta \mathbf{E}_{h}^{n} \cdot \mathbf{E}_{h}^{n} d x+\int_{\Omega} \mu^{-1} \delta \mathbf{B}_{h}^{n} \cdot \mathbf{B}_{h}^{n} d x+\varphi^{n}\left(\mathbf{E}_{h}^{n}\right)=\int_{\Omega} \mathbf{f}^{n} \cdot \mathbf{E}_{h}^{n} d x \quad \forall n \in\{1, \ldots, N\} .
$$

Now, fix $i_{0} \in\{1, \ldots, N\}$ and sum (3.17) up over $\left\{1, \ldots, i_{0}\right\}$. Then, applying the binomial formulas along with the Hölder and Young inequalities, we deduce that

$$
\begin{gathered}
\left\|\mathbf{E}_{h}^{i_{0}}\right\|_{\mathbf{L}_{\epsilon}^{2}(\Omega)}^{2}+\left\|\mathbf{B}_{h}^{i_{0}}\right\|_{\mathbf{L}_{1 / \mu}^{2}(\Omega)}+\sum_{n=1}^{i_{0}}\left\|\mathbf{E}_{h}^{n}-\mathbf{E}_{h}^{n-1}\right\|_{\mathbf{L}_{\epsilon}^{2}(\Omega)}^{2}+\sum_{n=1}^{i_{0}}\left\|\mathbf{B}_{h}^{n}-\mathbf{B}_{h}^{n-1}\right\|_{\mathbf{L}_{1 / \mu}^{2}(\Omega)}^{2} \\
+\tau \sum_{n=1}^{i_{0}} \varphi^{n}\left(\mathbf{E}_{h}^{n}\right) \leq \frac{1}{2} \sum_{n=1}^{i_{0}} \tau\left\|\mathbf{f}^{n}\right\|_{\mathbf{L}^{2}(\Omega)}^{2}+\left\|\mathbf{E}_{0 h}\right\|_{\mathbf{L}_{\epsilon}^{2}(\Omega)}^{2}+\left\|\mathbf{B}_{0 h}\right\|_{\mathbf{L}_{1 / \mu}^{2}(\Omega)}^{2}+\frac{1}{2} \sum_{n=1}^{i_{0}} \tau\left\|\mathbf{E}_{h}^{n}\right\|_{\mathbf{L}_{\epsilon}^{2}(\Omega)}^{2} .
\end{gathered}
$$

This, combined with (A4) and Lemma 3.2, gives us an estimate of the form

$$
\left\|\mathbf{E}_{h}^{i_{0}}\right\|_{\mathbf{L}_{\epsilon}^{2}(\Omega)}^{2} \leq C+\sum_{n=1}^{i_{0}-1} \tau\left\|\mathbf{E}_{h}^{n}\right\|_{\mathbf{L}_{\epsilon}^{2}(\Omega)}^{2} \quad \Rightarrow \quad\left\|\mathbf{E}_{h}^{i_{0}}\right\|_{\mathbf{L}_{\epsilon}^{2}(\Omega)}^{2} \leq C \exp \left(\sum_{n=1}^{i_{0}-1} \tau\right) \leq C,
$$

where we have used the discrete Gronwall inequality. Since $i_{0}$ was arbitrary, we see from (3.18) that the proof is finished.

Lemma 3.6. Let Assumptions 2.1, 2.2, and 3.1 hold. Then, there exists a constant $C>0$, depending only on $\mathbf{f}, \mathbf{E}_{0}, \mathbf{B}_{0}$ and $T, \epsilon, \mu$, such that for every $N \in \mathbb{N}$ and $h>0$ the solution $\left\{\left(\mathbf{E}_{h}^{n}, \mathbf{B}_{h}^{n}\right)\right\}_{n=1}^{N}$ of $\left(\mathrm{VI}_{N, h}\right)$ satisfies

$$
\begin{aligned}
\max _{n \in\{1, \ldots, N\}}\left\|\delta \mathbf{E}_{h}^{n}\right\|_{\mathbf{L}_{\epsilon}^{2}(\Omega)}^{2}+\max _{n \in\{1, \ldots, N\}}\left\|\delta \mathbf{B}_{h}^{n}\right\|_{\mathbf{L}_{1 / \mu}^{2}(\Omega)}^{2} \\
+\sum_{n=1}^{N}\left\|\delta \mathbf{E}_{h}^{n}-\delta \mathbf{E}_{h}^{n-1}\right\|_{\mathbf{L}_{\epsilon}^{2}(\Omega)}^{2}+\sum_{n=1}^{N}\left\|\delta \mathbf{B}_{h}^{n}-\delta \mathbf{B}_{h}^{n-1}\right\|_{\mathbf{L}_{1 / \mu}^{2}(\Omega)}^{2} \leq C
\end{aligned}
$$

and

$$
\max _{n \in\{1, \ldots, N\}}\left\|\operatorname{curl} \mathbf{E}_{h}^{n}\right\|_{\mathbf{L}^{2}(\Omega)}^{2} \leq C .
$$

Proof. Fix $n \in\{1, \ldots, N\}$. Again, inserting $\mathbf{v}_{h}=\mathbf{E}_{h}^{n}$ in the $n$-th inequality of $\left(\mathrm{VI}_{N, h}\right)$ and subtracting the $(n-1)$-th inequality tested with $\mathbf{v}_{h}=\mathbf{E}_{h}^{n-1}$ leads to

$$
\int_{\Omega} \mu^{-1}\left(\delta \mathbf{B}_{h}^{n}-\delta \mathbf{B}_{h}^{n-1}\right) \cdot \mathbf{w}_{h} d x+\int_{\Omega} \mu^{-1} \operatorname{curl}\left(\mathbf{E}_{h}^{n}-\mathbf{E}_{h}^{n-1}\right) \cdot \mathbf{w}_{h} d x=0 \quad \forall \mathbf{w}_{h} \in \mathbf{W}_{h} .
$$

We sum up (3.13) and (3.21) to conclude after setting $\left(\mathbf{v}_{h}, \mathbf{w}_{h}\right)=\left(\delta \mathbf{E}_{h}^{n}, \delta \mathbf{B}_{h}^{n}\right)$ that

$$
\begin{aligned}
\int_{\Omega} \epsilon\left(\delta \mathbf{E}_{h}^{n}-\delta \mathbf{E}_{h}^{n-1}\right) \cdot \delta \mathbf{E}_{h}^{n} d x+\int_{\Omega} \mu^{-1}\left(\delta \mathbf{B}_{h}^{n}-\delta \mathbf{B}_{h}^{n-1}\right) \cdot \delta \mathbf{B}_{h}^{n} d x & \\
& =\int_{\Omega}\left(\mathbf{f}^{n}-\mathbf{f}^{n-1}\right) \cdot \delta \mathbf{E}_{h}^{n} d x .
\end{aligned}
$$


Since (3.22) has a similar structure as (3.17), the arguments used in the proof of Lemma 3.5 are applicable to obtain (3.19). Finally, (3.20) follows immediately from (3.7) and (3.19).

With these stability results at hand and the discrete Gauss law for $\left(\mathrm{VI}_{N, h}\right)$, we are able to prove strong convergence of $\left\{\left(\mathbf{E}_{h}^{n}, \mathbf{B}_{h}^{n}\right)\right\}_{n=1}^{N}$ towards the unique solution of (VI). First, we denote

$$
\left\{\begin{array} { l } 
{ \mathbf { E } _ { N , h } ( 0 ) : = \mathbf { E } _ { 0 h } } \\
{ \mathbf { E } _ { N , h } ( t ) : = \mathbf { E } _ { h } ^ { n - 1 } + ( t - t _ { n - 1 } ) \delta \mathbf { E } _ { h } ^ { n } }
\end{array} \quad \text { and } \quad \left\{\begin{array}{l}
\overline{\mathbf{E}}_{N, h}(0):=\mathbf{E}_{0 h} \\
\overline{\mathbf{E}}_{N, h}(t):=\mathbf{E}_{h}^{n}
\end{array}\right.\right.
$$

for $t \in\left(t_{n-1}, t_{n}\right]$ and $n \in\{1, \ldots, N\}$. In the same way, we define $\mathbf{B}_{N, h}, \overline{\mathbf{B}}_{N, h}$ and $\overline{\mathbf{f}}_{N}$. Furthermore, we introduce the function $\varphi_{N}:[0, T] \times \mathbf{L}^{2}(\Omega) \rightarrow \mathbb{R}$ by

$$
\left\{\begin{array}{l}
\varphi_{N}(0, \mathbf{v}):=\varphi(\theta(0), \mathbf{v})=\int_{\Omega} j_{c}(x, \theta(x, 0))|\mathbf{v}(x)| d x \\
\varphi_{N}(t, \mathbf{v}):=\varphi^{n}(\mathbf{v})=\int_{\Omega} j_{c}\left(x, \theta\left(x, t_{n}\right)\right)|\mathbf{v}(x)| d x \quad \forall t \in\left(t_{n-1}, t_{n}\right],
\end{array}\right.
$$

for $\mathbf{v} \in \mathbf{L}^{2}(\Omega)$ and $n \in\{1, \ldots, N\}$. Now, we can rewrite $\left(\mathrm{VI}_{N, h}\right)$ in the following manner:

\section{$\left(\mathrm{VI}_{N, h}\right)$}

$$
\left\{\begin{array}{l}
\int_{\Omega} \epsilon \partial_{t} \mathbf{E}_{N, h}(t) \cdot\left(\mathbf{v}_{h}-\overline{\mathbf{E}}_{N, h}(t)\right)+\mu^{-1} \partial_{t} \mathbf{B}_{N, h}(t) \cdot\left(\mathbf{w}_{h}-\overline{\mathbf{B}}_{N, h}(t)\right) d x \\
\quad+\int_{\Omega} \mu^{-1} \operatorname{curl} \overline{\mathbf{E}}_{N, h}(t) \cdot \mathbf{w}_{h}-\mu^{-1} \overline{\mathbf{B}}_{N, h}(t) \cdot \operatorname{curl} \mathbf{v}_{h} d x \\
\quad+\varphi_{N}\left(t, \mathbf{v}_{h}\right)-\varphi_{N}\left(t, \overline{\mathbf{E}}_{N, h}(t)\right) \geq \int_{\Omega} \overline{\mathbf{f}}_{N}(t) \cdot\left(\mathbf{v}_{h}-\overline{\mathbf{E}}_{N, h}(t)\right) d x \\
\quad \text { for every }\left(\mathbf{v}_{h}, \mathbf{w}_{h}\right) \in \mathbf{V}_{h} \times \mathbf{W}_{h} \text { and a.e. } t \in(0, T)
\end{array}\right.
$$

$$
\left(\mathbf{E}_{N, h}(0), \mathbf{B}_{N, h}(0)\right)=\left(\mathbf{E}_{0 h}, \mathbf{B}_{0 h}\right) .
$$

The following discrete compactness result is well-known (cf. [14] for the original result with $\epsilon \equiv 1)$ and serves as a helpful tool to prove the strong convergence of $\left(\mathrm{VI}_{N, h}\right)$.

Lemma 3.7 ([28, Lemma 4.4]). Let $\left\{\mathbf{z}_{h}\right\}_{h>0} \subset \mathbf{H}_{0}$ (curl) be bounded and satisfy $\mathbf{z}_{h} \in \mathbf{X}_{h}^{(\epsilon)}$ for all $h>0$. Then, there exists a subsequence $\left\{\mathbf{z}_{h_{n}}\right\}_{n=1}^{\infty} \subset\left\{\mathbf{z}_{h}\right\}_{h>0}$ with $h_{n} \rightarrow 0$ as $n \rightarrow \infty$ such that

$$
\begin{aligned}
\mathbf{z}_{h_{n}} & \rightarrow \mathbf{z} & & \text { in } \mathbf{L}^{2}(\Omega) \text { as } n \rightarrow \infty \\
\operatorname{curl} \mathbf{z}_{h_{n}} & \rightarrow \mathbf{c u r l} \mathbf{z} & & \text { weakly in } \mathbf{L}^{2}(\Omega) \text { as } n \rightarrow \infty
\end{aligned}
$$

for some $\mathbf{z} \in \mathbf{X}^{(\epsilon)}(\Omega)$.

TheOREm 3.8. Let Assumptions 2.1, 2.2, and 3.1 hold. Then, there exists a pair

$$
(\mathbf{E}, \mathbf{B}) \in W^{1, \infty}\left((0, T), \mathbf{L}_{\epsilon}^{2}(\Omega) \times \mathbf{H}_{0}(\operatorname{div}=0)\right) \cap L^{\infty}\left((0, T), \mathbf{X}^{(\epsilon)}(\Omega) \times \mathbf{H}_{0}(\operatorname{div}=0)\right)
$$

such that for $N=N(h)$ with $N(h) \rightarrow \infty$ as $h \rightarrow 0$

$$
\begin{aligned}
& \lim _{h \rightarrow 0}\left\|\mathbf{E}_{N, h}-\mathbf{E}\right\|_{\mathcal{C}\left([0, T], \mathbf{L}_{\epsilon}^{2}(\Omega)\right)}+\left\|\mathbf{B}_{N, h}-\mathbf{B}\right\|_{\mathcal{C}\left([0, T], \mathbf{L}_{1 / \mu}^{2}(\Omega)\right)}=0, \\
& \lim _{h \rightarrow 0}\left\|\overline{\mathbf{E}}_{N, h}-\mathbf{E}\right\|_{L^{\infty}\left((0, T), \mathbf{L}_{\epsilon}^{2}(\Omega)\right)}+\left\|\overline{\mathbf{B}}_{N, h}-\mathbf{B}\right\|_{L^{\infty}\left((0, T), \mathbf{L}_{1 / \mu}^{2}(\Omega)\right)}=0,
\end{aligned}
$$

and $(\mathbf{E}, \mathbf{B})$ is the unique solution to $(\mathrm{VI})$. 
Proof. First of all, we emphasize that $N=N(h)$ denotes a family of natural numbers with $N(h) \rightarrow \infty$ for $h \rightarrow 0$. As shown in Lemma 3.5 and Lemma 3.6, $\left\{\mathbf{E}_{N, h}\right\}_{h>0},\left\{\mathbf{B}_{N, h}\right\}_{h>0},\left\{\overline{\mathbf{E}}_{N, h}\right\}_{h>0},\left\{\overline{\mathbf{B}}_{N, h}\right\}_{h>0}$, and $\left\{\partial_{t} \mathbf{E}_{N, h}\right\}_{h>0},\left\{\partial_{t} \mathbf{B}_{N, h}\right\}_{h>0}$ are bounded in their respective spaces. Therefore, we may extract weakly-* converging subsequences, which will not be denoted in a special way:

$$
\left\{\begin{aligned}
\overline{\mathbf{E}}_{N, h} \rightarrow^{*} \overline{\mathbf{E}} & \text { weakly-* in } L^{\infty}\left((0, T), \mathbf{H}_{0}(\mathbf{c u r l})\right), \\
\overline{\mathbf{B}}_{N, h} \rightarrow^{*} \overline{\mathbf{B}} & \text { weakly-* in } L^{\infty}\left((0, T), \mathbf{H}_{0}(\operatorname{div}=0)\right), \\
\mathbf{E}_{N, h} \rightarrow^{*} \mathbf{E} & \text { weakly-* in } L^{\infty}\left((0, T), \mathbf{H}_{0}(\mathbf{c u r l})\right), \\
\mathbf{B}_{N, h} \rightarrow^{*} \mathbf{B} & \text { weakly-* in } L^{\infty}\left((0, T), \mathbf{H}_{0}(\operatorname{div}=0)\right), \\
\partial_{t} \mathbf{E}_{N, h} \rightarrow^{*} \xi & \text { weakly-* in } L^{\infty}\left((0, T), \mathbf{L}_{\epsilon}^{2}(\Omega)\right), \\
\partial_{t} \mathbf{B}_{N, h} \rightarrow^{*} \chi & \text { weakly-* in } L^{\infty}\left((0, T), \mathbf{H}_{0}(\operatorname{div}=0)\right),
\end{aligned}\right.
$$

for some $\mathbf{E}, \mathbf{B}, \overline{\mathbf{E}}, \overline{\mathbf{B}}, \xi, \chi$ as $h \rightarrow 0$. First of all, we verify that $\mathbf{E}=\overline{\mathbf{E}}$ and $\mathbf{B}=\overline{\mathbf{B}}$. However, this is readily seen by the definition (3.23) and Lemma 3.6 since

$$
\begin{aligned}
& \left\|\overline{\mathbf{E}}_{N, h}-\mathbf{E}_{N, h}\right\|_{L^{\infty}\left((0, T), \mathbf{L}_{\epsilon}^{2}(\Omega)\right)} \leq \tau \max _{n \in\{1, \ldots, N\}}\left\|\delta \mathbf{E}_{h}^{n}\right\|_{\mathbf{L}_{\epsilon}^{2}(\Omega)} \leq C \tau \\
& \left\|\overline{\mathbf{B}}_{N, h}-\mathbf{B}_{N, h}\right\|_{L^{\infty}\left((0, T), \mathbf{L}_{1 / \mu}^{2}(\Omega)\right)} \leq \tau \max _{n \in\{1, \ldots, N\}}\left\|\delta \mathbf{B}_{h}^{n}\right\|_{\mathbf{L}_{1 / \mu}^{2}(\Omega)} \leq C \tau .
\end{aligned}
$$

Next, the classical integration by parts formula gives

$$
\begin{aligned}
& \int_{0}^{T}(\xi(t), \mathbf{v})_{\mathbf{L}_{\epsilon}^{2}(\Omega)} \phi(t) d t \underbrace{\leftarrow}_{(3.25)} \int_{0}^{T}\left(\partial_{t} \mathbf{E}_{N, h}(t), \mathbf{v}\right)_{\mathbf{L}_{\epsilon}^{2}(\Omega)} \phi(t) d t \\
&=-\int_{0}^{T}\left(\mathbf{E}_{N, h}(t), \mathbf{v}\right)_{\mathbf{L}_{\epsilon}^{2}(\Omega)} \phi^{\prime}(t) d t \underbrace{\rightarrow}_{(3.25)}-\int_{0}^{T}(\mathbf{E}(t), \mathbf{v})_{\mathbf{L}_{\epsilon}^{2}(\Omega)} \phi^{\prime}(t) d t
\end{aligned}
$$

for every $\mathbf{v} \in \mathbf{L}^{2}(\Omega)$ and $\phi \in \mathcal{C}_{0}^{\infty}(0, T)$, which yields $\xi=\partial_{t} \mathbf{E}$ and so

$$
\mathbf{E} \in W^{1, \infty}\left((0, T), \mathbf{L}_{\epsilon}^{2}(\Omega)\right) \cap L^{\infty}\left((0, T), \mathbf{H}_{0}(\mathbf{c u r l})\right) .
$$

Obviously, the same conclusion can be drawn for $\chi=\partial_{t} \mathbf{B}$, which implies that $\mathbf{B} \in$ $W^{1, \infty}\left((0, T), \mathbf{H}_{0}(\operatorname{div}=0)\right)$. Note that

$$
(\mathbf{E}, \mathbf{B}) \in W^{1, \infty}\left((0, T), \mathbf{L}_{\epsilon}^{2}(\Omega) \times \mathbf{L}_{1 / \mu}^{2}(\Omega)\right) \hookrightarrow \mathcal{C}\left([0, T], \mathbf{L}_{\epsilon}^{2}(\Omega) \times \mathbf{L}_{1 / \mu}^{2}(\Omega)\right)
$$

implies possibly after a modification on a subset of $[0, T]$ with measure zero that $(\mathbf{E}, \mathbf{B}) \in \mathcal{C}\left([0, T], \mathbf{L}_{\epsilon}^{2}(\Omega) \times \mathbf{L}_{1 / \mu}^{2}(\Omega)\right)$. Next, we prove the pointwise weak convergence

$$
\mathbf{E}_{N, h}(t) \rightarrow \mathbf{E}(t) \text { weakly in } \mathbf{L}_{\epsilon}^{2}(\Omega) \text { and } \mathbf{B}_{N, h}(t) \rightarrow \mathbf{B}(t) \text { weakly in } \mathbf{L}_{1 / \mu}^{2}(\Omega)
$$

for every $t \in[0, T]$. For that purpose, we fix $t \in(0, T], \mathbf{w} \in \mathbf{L}^{2}(\Omega)$ and $\phi \in \mathcal{C}^{1}([0, t])$. Then, integration by parts yields

$$
\begin{aligned}
& \text { (3.28) } \int_{0}^{t}\left(\partial_{t} \mathbf{E}(s), \mathbf{w}\right)_{\mathbf{L}_{\epsilon}^{2}(\Omega)} \phi(s) d s \leftarrow \int_{0}^{t}\left(\partial_{t} \mathbf{E}_{N, h}(s), \mathbf{w}\right)_{\mathbf{L}_{\epsilon}^{2}(\Omega)} \phi(s) d s \\
& =-\int_{0}^{t}\left(\mathbf{E}_{N, h}(s), \mathbf{w}\right)_{\mathbf{L}_{\epsilon}^{2}(\Omega)} \phi^{\prime}(s) d s+\left(\mathbf{E}_{N, h}(t), \mathbf{w}\right)_{\mathbf{L}_{\epsilon}^{2}(\Omega)} \phi(t)-\left(\mathbf{E}_{N, h}(0), \mathbf{w}\right)_{\mathbf{L}_{\epsilon}^{2}(\Omega)} \phi(0) .
\end{aligned}
$$


Choosing $\phi(0)=0$ as well as $\phi(t) \neq 0$ and applying integration by parts again gives

$$
\lim _{h \rightarrow 0}\left(\mathbf{E}_{N, h}(t), \mathbf{w}\right)_{\mathbf{L}_{\epsilon}^{2}(\Omega)}=(\mathbf{E}(t), \mathbf{w})_{\mathbf{L}_{\epsilon}^{2}(\Omega)} \quad \forall \mathbf{w} \in \mathbf{L}^{2}(\Omega) .
$$

Applying the above convergence to (3.28) and choosing $\phi(0) \neq 0$ leads to

$$
\lim _{h \rightarrow 0}\left(\mathbf{E}_{N, h}(0), \mathbf{w}\right)_{\mathbf{L}_{\epsilon}^{2}(\Omega)}=(\mathbf{E}(0), \mathbf{w})_{\mathbf{L}_{\epsilon}^{2}(\Omega)} \quad \forall \mathbf{w} \in \mathbf{L}^{2}(\Omega) .
$$

The same results hold also for $\mathbf{B}_{N, h}$, and so we conclude that (3.27) is valid.

Theorem 3.3, Lemma 3.5 and Lemma 3.6 imply that for every $t \in[0, T]$ the sequence $\left\{\mathbf{E}_{N, h}(t)\right\}_{h>0}$ is bounded in $\mathbf{H}_{0}(\mathbf{c u r l})$ and $\mathbf{E}_{N, h}(t) \in \mathbf{X}_{h}^{(\epsilon)}$ for all $h>0$. Thus, Lemma 3.7 and (3.27) yield that

$$
\lim _{h \rightarrow 0}\left\|\mathbf{E}_{N, h}(t)-\mathbf{E}(t)\right\|_{\mathbf{L}_{\epsilon}^{2}(\Omega)}=0 \quad \text { and } \quad \mathbf{E}(t) \in \mathbf{X}^{(\epsilon)}(\Omega) \quad \forall t \in[0, T] .
$$

In particular, thanks to Lemma 3.2, we have $\mathbf{E}(0)=\mathbf{E}_{0}$. By (3.29) and Lemma 3.6, the Arzelà-Ascoli theorem for Banach space-valued functions (cf. [15, Theorem 3.1]) implies that

$$
\lim _{h \rightarrow 0}\left\|\mathbf{E}_{N, h}-\mathbf{E}\right\|_{\mathcal{C}\left([0, T], \mathbf{L}_{\epsilon}^{2}(\Omega)\right)}=0 \underbrace{\Rightarrow}_{(3.26)} \lim _{h \rightarrow 0}\left\|\overline{\mathbf{E}}_{N, h}-\mathbf{E}\right\|_{L^{\infty}\left((0, T), \mathbf{L}_{\epsilon}^{2}(\Omega)\right)}=0 .
$$

We continue and recall the classical identity:

$$
\int_{0}^{t}\left(\partial_{t} \mathbf{B}_{N, h}(s), \mathbf{B}_{N, h}(s)\right)_{\mathbf{L}_{1 / \mu}^{2}(\Omega)} d s=\frac{1}{2}\left\|\mathbf{B}_{N, h}(t)\right\|_{\mathbf{L}_{1 / \mu}^{2}(\Omega)}^{2}-\frac{1}{2}\left\|\mathbf{B}_{0 h}\right\|_{\mathbf{L}_{1 / \mu}^{2}(\Omega)}^{2} .
$$

Combining (3.31) with (3.27) and Lemma 3.2 yields

$$
\begin{aligned}
& \liminf _{h \rightarrow 0} \int_{0}^{t}\left(\partial_{t} \mathbf{B}_{N, h}(s), \overline{\mathbf{B}}_{N, h}(s)\right)_{\mathbf{L}_{1 / \mu}^{2}(\Omega)} d s \\
& \underbrace{=}_{(3.26)} \liminf _{h \rightarrow 0} \int_{0}^{t}\left(\partial_{t} \mathbf{B}_{N, h}(s), \mathbf{B}_{N, h}(s)\right)_{\mathbf{L}_{1 / \mu}^{2}(\Omega)} d s \\
& \underbrace{=}_{(3.31)} \liminf _{h \rightarrow 0} \frac{1}{2}\left\|\mathbf{B}_{N, h}(t)\right\|_{\mathbf{L}_{1 / \mu}^{2}(\Omega)}^{2}-\frac{1}{2}\left\|\mathbf{B}_{0 h}\right\|_{\mathbf{L}_{1 / \mu}^{2}(\Omega)}^{2} \\
& \geq \frac{1}{2}\|\mathbf{B}(t)\|_{\mathbf{L}_{1 / \mu}^{2}(\Omega)}^{2}-\frac{1}{2}\|\mathbf{B}(0)\|_{\mathbf{L}_{1 / \mu}^{2}}^{2}=\frac{1}{2} \int_{0}^{t}\left(\partial_{t} \mathbf{B}(s), \mathbf{B}(s)\right)_{\mathbf{L}_{1 / \mu}^{2}(\Omega)} d s,
\end{aligned}
$$

where the above inequality holds due to the fact that the squared norm is weakly lower semicontinuous. Next, we prove the pointwise convergence

$$
\lim _{h \rightarrow 0} \varphi_{N}\left(t, \overline{\mathbf{E}}_{N, h}(t)\right)-\varphi(\theta(t), \mathbf{E}(t))=0 \quad \forall t \in[0, T] .
$$

For $t=0$, (3.33) follows immediately from (3.23)-(3.24) and Lemma 3.2. Let now $t \in(0, T]$. Then, for every $N \in \mathbb{N}$, there exists a unique $n \in\{1, \ldots, N\}$ such that $t \in\left(t_{n-1}, t_{n}\right]$. Hence, the sequence $\tilde{t}_{N, h}:=t_{n}$ fulfills $\tilde{t}_{N, h} \rightarrow t$ as $h \rightarrow 0$. Making use of this sequence, we obtain that

(3.34) $\left|\varphi_{N}\left(t, \overline{\mathbf{E}}_{N, h}(t)\right)-\varphi(\theta(t), \mathbf{E}(t))\right| \leq$

$$
\int_{\Omega} j_{c}\left(x, \theta\left(x, \tilde{t}_{N, h}\right)\right)\left|\overline{\mathbf{E}}_{N, h}(t)-\mathbf{E}(t)\right| d x+\int_{\Omega}\left|j_{c}\left(x, \theta\left(x, \tilde{t}_{N, h}\right)\right)-j_{c}(x, \theta(x, t))\right||\mathbf{E}(t)| d x
$$

$\underbrace{\leq}_{(A 3)-(A 4)} C\left\|\overline{\mathbf{E}}_{N, h}-\mathbf{E}\right\|_{L^{\infty}\left((0, T), \mathbf{L}^{2}(\Omega)\right)}+\int_{\Omega}\left|j_{c}\left(x, \theta\left(x, \tilde{t}_{N, h}\right)\right)-j_{c}(x, \theta(x, t)) \| \mathbf{E}(t)\right| d x$. 
As shown in (3.30), the first term in the right-hand side of (3.34) converges to 0 as $h \rightarrow 0$. In order to pass to the limit in the second term, we make use of (A2) and (A4) to deduce after selecting a subsequence that

$$
\lim _{h \rightarrow 0} j_{c}\left(x, \theta\left(x, \tilde{t}_{N, h}\right)\right)-j_{c}(x, \theta(x, t))=0 \quad \text { for a.e. } x \in \Omega,
$$

and so, thanks to (A3)-(A4), Lebesgue's theorem yields

$$
\lim _{h \rightarrow 0} \int_{\Omega}\left|j_{c}\left(x, \theta\left(\tilde{t}_{N, h}, x\right)\right)-j_{c}(x, \theta(t, x))\right||\mathbf{E}(t)| d x=0 .
$$

In conclusion, (3.33) is valid.

Now, we show that $(\mathbf{E}, \mathbf{B})$ is a solution to (VI): Fix $t \in(0, T], \tilde{h}>0$, integrate $\left(\mathrm{VI}_{N, h}\right)$ for $h<\tilde{h}$ over $[0, t]$ and test it with $\left(\mathbf{v}_{\tilde{h}}, \mathbf{w}_{\tilde{h}}\right) \in \mathbf{V}_{\tilde{h}} \times \mathbf{W}_{\tilde{h}}$. Afterwards, we apply the limes superior to the resulting system to deduce that

$$
\int_{0}^{t}\left(\mathbf{f}(s), \mathbf{v}_{\tilde{h}}-\mathbf{E}(s)\right)_{\mathbf{L}^{2}(\Omega)} d s=\limsup _{h \rightarrow 0} \int_{0}^{t}\left(\overline{\mathbf{f}}_{N}(s), \mathbf{v}_{\tilde{h}}-\overline{\mathbf{E}}_{N, h}(s)\right)_{\mathbf{L}^{2}(\Omega)} d s
$$

$$
\underbrace{\leq}_{\left(\mathrm{VI}_{N, h}\right)} \limsup _{h \rightarrow 0}\left[\int_{0}^{t}\left(\partial_{t} \mathbf{E}_{N, h}(s), \mathbf{v}_{\tilde{h}}-\overline{\mathbf{E}}_{N, h}(s)\right)_{\mathbf{L}_{\epsilon}^{2}(\Omega)} d s\right.
$$$$
+\int_{0}^{t}\left(\partial_{t} \mathbf{B}_{N, h}(s), \mathbf{w}_{\tilde{h}}-\overline{\mathbf{B}}_{N, h}(s)\right)_{\mathbf{L}_{1 / \mu}^{2}(\Omega)} d s+\int_{0}^{t}\left(\operatorname{curl} \overline{\mathbf{E}}_{N, h}(s), \mathbf{w}_{\tilde{h}}\right)_{\mathbf{L}_{1 / \mu}^{2}(\Omega)} d s
$$$$
\left.-\int_{0}^{t}\left(\overline{\mathbf{B}}_{N, h}(s), \operatorname{curl} \mathbf{v}_{\tilde{h}}\right)_{\mathbf{L}_{1 / \mu}^{2}(\Omega)} d s+\int_{0}^{t} \varphi_{N}\left(s, \mathbf{v}_{\tilde{h}}\right)-\varphi_{N}\left(s, \overline{\mathbf{E}}_{N, h}(s)\right) d s\right]
$$$$
\leq \int_{0}^{t}\left(\partial_{t} \mathbf{E}(s), \mathbf{v}_{\tilde{h}}-\mathbf{E}(s)\right)_{\mathbf{L}_{\epsilon}^{2}(\Omega)}+\left(\partial_{t} \mathbf{B}(s), \mathbf{w}_{\tilde{h}}-\mathbf{B}(s)\right)_{\mathbf{L}_{1 / \mu}^{2}(\Omega)} d s
$$$$
+\int_{0}^{t}\left(\operatorname{curl} \mathbf{E}(s), \mathbf{w}_{\tilde{h}}\right)_{\mathbf{L}_{1 / \mu}^{2}(\Omega)}-\left(\mathbf{B}(s), \operatorname{curl} \mathbf{v}_{\tilde{h}}\right)_{\mathbf{L}_{1 / \mu}^{2}(\Omega)} d s
$$$$
+\int_{0}^{t} \varphi\left(\theta(s), \mathbf{v}_{\tilde{h}}\right)-\varphi(\theta(s), \mathbf{E}(s)) d s .
$$

Obviously, (3.25) was used to get the above estimate. Furthermore, we employed (3.30) to obtain convergence of the first term and (3.32) to estimate the second limit. Ultimately, we combined (3.33) with Lebesgue's theorem to obtain convergence of the last time-integral. Since there is no restriction to $\tilde{h}>0$, the density of $\mathbf{V}_{h} \subset$ $\mathbf{H}_{0}$ (curl) and $\mathbf{W}_{h} \subset \mathbf{L}^{2}(\Omega)$ yields, if we differentiate (3.36) with respect to $t$, that $(\mathbf{E}, \mathbf{B}) \in W^{1, \infty}\left((0, T), \mathbf{L}_{\epsilon}^{2}(\Omega) \times \mathbf{H}_{0}(\operatorname{div}=0)\right) \cap L^{\infty}\left((0, T), \mathbf{X}^{(\epsilon)}(\Omega) \times \mathbf{H}_{0}(\operatorname{div}=0)\right)$ satisfies the evolutionary variational inequality $(\mathrm{VI})$.

The uniqueness of the solution to (VI) follows by an energy argument: For $t \in$ $(0, T]$ testing of $(\mathrm{VI})$ with $(\mathbf{v}, \mathbf{w})=(2 \mathbf{E}(t), 2 \mathbf{B}(t))$ and $(\mathbf{v}, \mathbf{w})=(0,0)$ leads to

$$
\int_{\Omega} \epsilon \partial_{t} \mathbf{E}(t) \cdot \mathbf{E}(t) d x+\int_{\Omega} \mu^{-1} \partial_{t} \mathbf{B}(t) \cdot \mathbf{B}(t) d x+\varphi(\theta(t), \mathbf{E}(t))=\int_{\Omega} \mathbf{f}(t) \cdot \mathbf{E}(t) d x .
$$

The combination of (VI) with (3.37) yields

$$
\begin{aligned}
& \int_{\Omega} \epsilon \partial_{t} \mathbf{E}(t) \cdot \mathbf{v} d x+\int_{\Omega} \mu^{-1} \partial_{t} \mathbf{B}(t) \cdot \mathbf{w} d x+\int_{\Omega} \mu^{-1} \operatorname{curl} \mathbf{E}(t) \cdot \mathbf{w} d x \\
&-\int_{\Omega} \mu^{-1} \mathbf{B}(t) \cdot \operatorname{curl} \mathbf{v} d x+\varphi(\theta(t), \mathbf{v}) \geq \int_{\Omega} \mathbf{f}(t) \cdot \mathbf{v} d x
\end{aligned}
$$


for every $(\mathbf{v}, \mathbf{w}) \in \mathbf{H}_{0}(\mathbf{c u r l}) \times \mathbf{L}^{2}(\Omega)$. Let $(\tilde{\mathbf{E}}, \tilde{\mathbf{B}}) \in W^{1, \infty}\left((0, T), \mathbf{L}_{\epsilon}^{2}(\Omega) \times \mathbf{L}_{\mu}^{2}(\Omega)\right) \cap$ $L^{\infty}\left((0, T), \mathbf{H}_{0}(\mathbf{c u r l}) \times \mathbf{L}_{\mu}^{2}(\Omega)\right)$ be another solution to (VI). Again, testing of (VI) with $(\mathbf{v}, \mathbf{w})=(2 \tilde{\mathbf{E}}(t), 2 \tilde{\mathbf{B}}(t))$ and $(\mathbf{v}, \mathbf{w})=(0,0)$, leads to the fact that $(\tilde{\mathbf{E}}, \tilde{\mathbf{B}})$ satisfies (3.38), with $(\mathbf{E}, \mathbf{B})$ replaced by $(\tilde{\mathbf{E}}, \tilde{\mathbf{B}})$. For this reason, the difference $(\mathbf{e}, \mathbf{b}):=(\mathbf{E}-$ $\tilde{\mathbf{E}}, \mathbf{B}-\tilde{\mathbf{B}})$ fulfills

$$
\begin{aligned}
\int_{\Omega} \epsilon \partial_{t} \mathbf{e}(t) \cdot \mathbf{v} d x & +\int_{\Omega} \mu^{-1} \partial_{t} \mathbf{b}(t) \cdot \mathbf{w} d x+\int_{\Omega} \mu^{-1} \operatorname{curl} \mathbf{e}(t) \cdot \mathbf{w} d x \\
& -\int_{\Omega} \mu^{-1} \mathbf{b}(t) \cdot \operatorname{curl} \mathbf{v} d x \geq 0 \quad \forall(\mathbf{v}, \mathbf{w}) \in \mathbf{H}_{0}(\mathbf{c u r l}) \times \mathbf{L}^{2}(\Omega) .
\end{aligned}
$$

Now, inserting $(\mathbf{v}, \mathbf{w})=(-\mathbf{e},-\mathbf{b})$ in $(3.39)$ gives us

$$
\frac{1}{2} \frac{d}{d t}\|\mathbf{e}(t)\|_{\mathbf{L}_{\epsilon}^{2}(\Omega)}^{2}+\frac{1}{2} \frac{d}{d t}\|\mathbf{b}(t)\|_{\mathbf{L}_{1 / \mu}^{2}(\Omega)}^{2} \leq 0 .
$$

Since $\mathbf{e}(0)=\mathbf{b}(0)=0$, the above inequality yields that $\mathbf{e}(t)=\mathbf{b}(t)=0$ for all $t \in[0, T]$. This implies that $(\mathbf{E}, \mathbf{B})$ is the unique solution to (VI).

We complete the proof by verifying the strong convergence of $\mathbf{B}_{N, h}$ towards $\mathbf{B}$. Recalling from (3.17), it holds that

$$
\begin{array}{r}
\int_{\Omega} \epsilon \partial_{t} \mathbf{E}_{N, h}(t) \cdot \overline{\mathbf{E}}_{N, h}(t) d x+\int_{\Omega} \mu^{-1} \partial_{t} \mathbf{B}_{N, h}(t) \cdot \overline{\mathbf{B}}_{N, h}(t) d x+\varphi_{N}\left(t, \overline{\mathbf{E}}_{N, h}(t)\right) \\
=\int_{\Omega} \overline{\mathbf{f}}_{N}(t) \cdot \overline{\mathbf{E}}_{N, h}(t) d x
\end{array}
$$

After subtraction of (3.37) and (3.40), integration over $(0, t)$ for $t \in(0, T]$, we obtain

$$
\begin{aligned}
& \int_{0}^{t}\left(\partial_{t} \mathbf{E}_{N, h}(s), \overline{\mathbf{E}}_{N, h}(s)\right)_{\mathbf{L}_{\epsilon}^{2}(\Omega)}-\left(\partial_{t} \mathbf{E}(s), \mathbf{E}(s)\right)_{\mathbf{L}_{\epsilon}^{2}(\Omega)} d s \\
& +\int_{0}^{t}\left(\partial_{t} \mathbf{B}_{N, h}(s), \overline{\mathbf{B}}_{N, h}(s)\right)_{\mathbf{L}_{1 / \mu}^{2}(\Omega)}-\left(\partial_{t} \mathbf{B}(s), \mathbf{B}(s)\right)_{\mathbf{L}_{1 / \mu}^{2}(\Omega)} d s \\
& +\int_{0}^{t} \varphi_{N}\left(s, \overline{\mathbf{E}}_{N, h}(s)\right)-\varphi(\theta(s), \mathbf{E}(s)) d s \\
= & \int_{0}^{t}\left(\overline{\mathbf{f}}_{N}(s), \overline{\mathbf{E}}_{N, h}(s)\right)_{\mathbf{L}^{2}(\Omega)}-(\mathbf{f}(s), \mathbf{E}(s))_{\mathbf{L}^{2}(\Omega)} d s .
\end{aligned}
$$

In view of (3.25) and (3.30), we get

$$
\int_{0}^{t}\left(\partial_{t} \mathbf{E}_{N, h}(s), \overline{\mathbf{E}}_{N, h}(s)\right)_{\mathbf{L}_{\epsilon}^{2}(\Omega)} d s-\int_{0}^{t}\left(\partial_{t} \mathbf{E}(s), \mathbf{E}(s)\right)_{\mathbf{L}_{\epsilon}^{2}(\Omega)} d s \rightarrow 0 \text { as } h \rightarrow 0 .
$$

The convergence of the right-hand side in (3.41) to 0 as $h \rightarrow 0$ also follows from (3.30). Now, simple algebraic rearrangements give

$$
\begin{aligned}
& \int_{\Omega} \mu^{-1} \partial_{t} \mathbf{B}_{N, h}(t) \cdot \overline{\mathbf{B}}_{N, h}(t) d x-\int_{\Omega} \mu^{-1} \partial_{t} \mathbf{B}(t) \cdot \mathbf{B}(t) d x \\
= & \int_{\Omega} \mu^{-1}\left(\partial_{t} \mathbf{B}_{N, h}(t)-\partial_{t} \mathbf{B}(t)\right) \cdot\left(\mathbf{B}_{N, h}(t)-\mathbf{B}(t)\right) d x \\
& +\int_{\Omega} \mu^{-1} \partial_{t} \mathbf{B}_{N, h}(t) \cdot\left(\overline{\mathbf{B}}_{N, h}(t)-\mathbf{B}_{N, h}(t)\right) d x+\int_{\Omega} \mu^{-1} \partial_{t} \mathbf{B}_{N, h}(t) \cdot \mathbf{B}(t) d x \\
& +\int_{\Omega} \mu^{-1} \partial_{t} \mathbf{B}(t) \cdot \mathbf{B}_{N, h}(t) d x-2 \int_{\Omega} \mu^{-1} \partial_{t} \mathbf{B}(t) \cdot \mathbf{B}(t) d x
\end{aligned}
$$


and the same identity used in (3.31) implies

$$
\begin{aligned}
& \int_{\Omega} \mu^{-1}\left(\partial_{t} \mathbf{B}_{N, h}(t)-\partial_{t} \mathbf{B}(t)\right) \cdot\left(\mathbf{B}_{N, h}(t)-\mathbf{B}(t)\right) d x \\
= & \frac{1}{2}\left\|\mathbf{B}_{N, h}(t)-\mathbf{B}(t)\right\|_{\mathbf{L}_{1 / \mu}^{2}(\Omega)}^{2}-\frac{1}{2}\left\|\mathbf{B}_{0 h}-\mathbf{B}_{0}\right\|_{\mathbf{L}_{1 / \mu}^{2}(\Omega)}^{2} .
\end{aligned}
$$

Applying (3.33) in combination with Lebesgue's dominated convergence theorem, (3.42)-(3.44), (3.25) and Lemma 3.2 to (3.41), we obtain after passing to the limit $h \rightarrow 0$ that

$$
\lim _{h \rightarrow 0}\left\|\mathbf{B}_{N, h}(t)-\mathbf{B}(t)\right\|_{\mathbf{L}_{1 / \mu}^{2}(\Omega)}=0 \quad \forall t \in[0, T] .
$$

In view of the above pointwise convergence and Lemma 3.6, the Arzelà-Ascoli theorem yields that

$$
\lim _{h \rightarrow 0}\left\|\mathbf{B}_{N, h}-\mathbf{B}\right\|_{\mathcal{C}\left([0, T], \mathbf{L}_{1 / \mu}^{2}(\Omega)\right)}=0 \underbrace{\Rightarrow}_{(3.26)} \lim _{h \rightarrow 0}\left\|\overline{\mathbf{B}}_{N, h}-\mathbf{B}\right\|_{L^{\infty}\left((0, T), \mathbf{L}_{1 / \mu}^{2}(\Omega)\right)}=0 .
$$

This completes the proof.

As a consequence, Theorem 3.8 yields the existence of a unique solution to (VI) obeying the physical Gauss law for electromagnetic fields:

Corollary 3.9. Let Assumptions 2.1, 2.2, and 3.1 hold. Then, (VI) admits a unique solution $(\mathbf{E}, \mathbf{B}) \in W^{1, \infty}\left((0, T), \mathbf{L}_{\epsilon}^{2}(\Omega) \times \mathbf{H}_{0}(\operatorname{div}=0)\right) \cap L^{\infty}\left((0, T), \mathbf{X}^{(\epsilon)}(\Omega) \times\right.$ $\left.\mathbf{H}_{0}(\operatorname{div}=0)\right)$.

4. A priori error analysis. We start by recalling the classical error estimates for the curl-conforming Nédélec interpolant $\mathcal{N}_{h}$ and the divergence-conforming Raviart-Thomas interpolant $\boldsymbol{\pi}_{h}$.

Lemma 4.1. Let $s \in\left(\frac{1}{2}, 1\right]$. There exists a constant $C>0$ independent of $h$ and $\mathbf{y}$, such that

$$
\begin{aligned}
\left\|\mathbf{y}-\mathcal{N}_{h} \mathbf{y}\right\|_{\mathbf{H}(\text { curl })} & \leq C h^{s}\|\mathbf{y}\|_{\mathbf{H}_{0}^{s}(\text { curl })} & & \forall \mathbf{y} \in \mathbf{H}_{0}^{s}(\text { curl }) \\
\left\|\mathbf{y}-\boldsymbol{\pi}_{h} \mathbf{y}\right\|_{\mathbf{L}^{2}(\Omega)} & \leq C h^{s}\|\mathbf{y}\|_{\mathbf{H}^{s}(\Omega)} & & \forall \mathbf{y} \in \mathbf{H}^{s}(\Omega)
\end{aligned}
$$

for all $h>0$. Here, $\mathbf{H}_{0}^{s}(\mathbf{c u r l}):=\left\{\mathbf{y} \in \mathbf{H}^{s}(\Omega) \cap \mathbf{H}_{0}(\operatorname{curl})\right.$ : $\left.\operatorname{curl} \mathbf{y} \in \mathbf{H}^{s}(\Omega)\right\}$.

These operators are extensively discussed in [21, Theorem 5.25, Theorem 5.41 and Remark 5.42]. For our a priori error analysis, we rely on the following higher regularity assumption:

Assumption 4.2 (Additional assumptions on the given data and the solution).

(A6) For every $M>0$, there exists a constant $L(M)>0$ such that

$$
\left|j_{c}(x, y)-j_{c}(x, z)\right| \leq L(M)|y-z|
$$

for a.e. $x \in \Omega$ and every $y, z \in[-M, M]$.

(A7) Suppose that $\theta \in \mathcal{C}^{0,1}\left([0, T], L^{2}(\Omega)\right) \cap \mathcal{C}\left([0, T], L^{\infty}(\Omega)\right)$.

(A8) There exists $s \in\left(\frac{1}{2}, 1\right]$ such that $\mathbf{E}_{0} \in \mathbf{X}_{h}^{(\epsilon)} \cap \mathbf{H}_{0}^{s}(\mathbf{c u r l})$ and $\mathbf{B}_{0} \in \mathbf{H}_{0}(\operatorname{div}=0) \cap$ $\mathbf{H}^{s}(\Omega)$.

(A9) The solution (E, B) of (VI) is assumed to satisfy

$$
\left\{\begin{array}{l}
\mathbf{E} \in L^{1}\left((0, T), \mathbf{H}_{0}^{s}(\mathbf{c u r l})\right), \\
\mathbf{B} \in L^{1}\left((0, T), \mathbf{H}^{s}(\Omega)\right) .
\end{array}\right.
$$


Assumption 4.2 gives a first error estimate for the initial value, which is proven by similar arguments, but in a much easier fashion than Theorem 4.4 and hence, we will not present it.

Lemma 4.3. Let Assumptions 2.1, 2.2, 3.1, and 4.2 hold. Then there exists a constant $C>0$, independent of $h>0$, such that

$$
\left\|\mathbf{E}_{0 h}-\mathbf{E}_{0}\right\|_{\mathbf{L}_{\epsilon}^{2}(\Omega)}^{2}+\left\|\mathbf{B}_{0 h}-\mathbf{B}_{0}\right\|_{\mathbf{L}_{1 / \mu}^{2}(\Omega)}^{2} \leq C h^{s} \quad \forall h>0 .
$$

Theorem 4.4. Let Assumptions 2.1, 2.2, 3.1, and 4.2 hold. Then, there exists a constant $C>0$, independent of $N \in \mathbb{N}$ and $h>0$, such that for all $t \in[0, T]$

$$
\begin{array}{r}
\left\|\mathbf{E}_{N, h}(t)-\mathbf{E}(t)\right\|_{\mathbf{L}_{\epsilon}^{2}(\Omega)}^{2}+\left\|\mathbf{B}_{N, h}(t)-\mathbf{B}(t)\right\|_{\mathbf{L}_{1 / \mu}^{2}(\Omega)}^{2} \leq C\left(h^{s}+\tau\right)\left(\|\mathbf{E}\|_{L^{1}\left((0, T), \mathbf{H}_{0}^{s}(\mathbf{c u r l})\right)}\right. \\
\left.+\left\|\partial_{t} \mathbf{E}\right\|_{L^{1}\left((0, T), \mathbf{L}_{\epsilon}^{2}(\Omega)\right)}+\|\mathbf{B}\|_{L^{1}\left((0, T), \mathbf{H}^{s}(\Omega)\right)}+\left\|\partial_{t} \mathbf{B}\right\|_{L^{1}\left((0, T), \mathbf{L}_{1 / \mu}^{2}(\Omega)\right)}+1\right)
\end{array}
$$

holds for every $h>0$ and every $N \in \mathbb{N}$.

Proof. We begin by testing (VI) with $(\mathbf{v}, \mathbf{w})=\left(\overline{\mathbf{E}}_{N, h}(t), \overline{\mathbf{B}}_{N, h}(t)\right)$ and $\left(\mathrm{VI}_{N, h}\right)$ with $\left(\mathbf{v}_{h}, \mathbf{w}_{h}\right)=\left(\mathcal{N}_{h} \mathbf{E}(t), \boldsymbol{\pi}_{h} \mathbf{B}(t)\right) \in \mathbf{V}_{h} \times \mathbf{W}_{h}$. Afterwards, fix $\sigma \in(0, T]$, summarize the resulting inequalities and integrate over $[0, \sigma]$ to obtain after some rearrangements

$$
\begin{aligned}
& \int_{0}^{\sigma} \int_{\Omega} \epsilon\left(\partial_{t} \mathbf{E}_{N, h}(t)-\partial_{t} \mathbf{E}(t)\right) \cdot\left(\overline{\mathbf{E}}_{N, h}(t)-\mathbf{E}(t)\right) d x d t \\
& \quad+\int_{0}^{\sigma} \int_{\Omega} \mu^{-1}\left(\partial_{t} \mathbf{B}_{N, h}(t)-\partial_{t} \mathbf{B}(t)\right) \cdot\left(\overline{\mathbf{B}}_{N, h}(t)-\mathbf{B}(t)\right) d x d t \\
& \leq \int_{0}^{\sigma}\left[\int_{\Omega} \overline{\mathbf{f}}_{N}(t) \cdot\left(\mathbf{E}(t)-\mathcal{N}_{h} \mathbf{E}(t)\right) d x+\int_{\Omega}\left(\mathbf{f}(t)-\overline{\mathbf{f}}_{N}(t)\right) \cdot\left(\mathbf{E}(t)-\overline{\mathbf{E}}_{N, h}(t)\right) d x\right. \\
& \quad+\int_{\Omega} \epsilon \partial_{t} \mathbf{E}_{N, h}(t) \cdot\left(\mathbf{E}(t)-\mathcal{N}_{h} \mathbf{E}(t)\right) d x+\int_{\Omega} \mu^{-1} \partial_{t} \mathbf{B}_{N, h}(t) \cdot\left(\mathbf{B}(t)-\boldsymbol{\pi}_{h} \mathbf{B}(t)\right) d x \\
& \quad+\int_{\Omega} \mu^{-1} \operatorname{curl} \mathbf{\mathbf { E }} \overline{\mathbf{E}}_{N, h}(t) \cdot\left(\boldsymbol{\pi}_{h} \mathbf{B}(t)-\mathbf{B}(t)\right)+\mu^{-1} \overline{\mathbf{B}}_{N, h}(s) \cdot \mathbf{c u r l}\left(\mathbf{E}(t)-\mathcal{N}_{h} \mathbf{E}(t)\right) d x \\
& \quad+\left(\varphi_{N}\left(t, \mathcal{N}_{h} \mathbf{E}(t)\right)-\varphi(\theta(t),(\mathbf{E}(t)))+\left(\varphi\left(\theta(t), \overline{\mathbf{E}}_{N, h}(t)\right)-\varphi_{N}\left(t, \overline{\mathbf{E}}_{N, h}(t)\right)\right)\right] d t \\
& =: \\
& \sum_{i=1}^{8} C_{i} .
\end{aligned}
$$

Next, we will give estimates for $C_{i}, i \in\{1, \ldots, 8\}$. The stability results in Lemma 3.5 and Lemma 3.6 combined with the regularity of (E, B) (see (A9)) as well as the error estimates for the interpolation operators in Lemma 4.1 let us easily deduce estimates for $C_{1}, C_{3}, C_{4}, C_{5}$ and $C_{6}$ as follows:

$$
\begin{aligned}
\left|C_{i}\right| & \leq C h^{s}\|\mathbf{E}\|_{L^{1}\left((0, T), \mathbf{H}_{0}^{s}(\mathbf{c u r l})\right)} \quad \forall i \in\{1,3,6\}, \\
\left|C_{i}\right| & \leq C h^{s}\|\mathbf{B}\|_{L^{1}\left((0, T), \mathbf{H}^{s}(\Omega)\right)} \quad \forall i \in\{4,5\} .
\end{aligned}
$$

To estimate $C_{2}$, we use the Lipschitz continuity of $\mathbf{f}$ (see (A4)) and Theorem 3.8 along with (3.26):

$$
\left|C_{2}\right| \leq C \tau \int_{0}^{\sigma}\left\|\mathbf{E}(t)-\overline{\mathbf{E}}_{N, h}(t)\right\|_{\mathbf{L}_{\epsilon}^{2}(\Omega)} d t \leq C \tau .
$$


Next, an estimate for $C_{7}$ is given: We begin with

$$
\begin{aligned}
& \left|\int_{0}^{\sigma} \varphi_{N}\left(t, \mathcal{N}_{h} \mathbf{E}(t)\right)-\varphi(\theta(t), \mathbf{E}(t)) d t\right| \\
\leq & \int_{0}^{T}\left|\varphi_{N}\left(t, \mathcal{N}_{h} \mathbf{E}(t)\right)-\varphi_{N}(t, \mathbf{E}(t))\right| d t+\int_{0}^{T}\left|\varphi_{N}(t, \mathbf{E}(t))-\varphi(\theta(t), \mathbf{E}(t))\right| d t .
\end{aligned}
$$

Because of (A3), the first term on the right-hand side of (4.6) satisfies

$$
\begin{aligned}
\int_{0}^{T}\left|\varphi_{N}\left(t, \mathcal{N}_{h} \mathbf{E}(t)\right)-\varphi_{N}(t, \mathbf{E}(t))\right| d t & \leq C \int_{0}^{T}\left\|\mathcal{N}_{h} \mathbf{E}(t)-\mathbf{E}(t)\right\|_{\mathbf{L}^{2}(\Omega)} d t \\
& \leq C h^{s}\|\mathbf{E}\|_{L^{1}\left((0, T), \mathbf{H}_{0}^{s}(\mathbf{c u r l})\right) .}
\end{aligned}
$$

On the other hand, the second term in (4.6) is estimated by using (A6) and (A7):

$$
\begin{aligned}
& \int_{0}^{T}\left|\varphi_{N}(t, \mathbf{E}(t))-\varphi(\theta(t), \mathbf{E}(t))\right| d t \\
\underbrace{=}_{(3.24)} & \sum_{n=1}^{N} \int_{t_{n-1}}^{t_{n}} \int_{\Omega}\left|j_{c}\left(x, \theta\left(t_{n}, x\right)\right)-j_{c}(x, \theta(t, x))\right||\mathbf{E}(t)| d x d t \\
\leq & C \sum_{n=1}^{N} \int_{t_{n-1}}^{t_{n}} \tau\|\mathbf{E}(t)\|_{\mathbf{L}^{2}(\Omega)} d t \leq C \tau\|\mathbf{E}\|_{L^{1}\left((0, T), \mathbf{L}_{\epsilon}^{2}(\Omega)\right)} .
\end{aligned}
$$

Combining (4.6)-(4.8), we obtain

$$
\left|C_{7}\right| \leq C\left(h^{s}+\tau\right)\|\mathbf{E}\|_{L^{1}\left((0, T), \mathbf{H}_{0}^{s}(\mathbf{c u r l})\right)} .
$$

We reuse the arguments from (4.8) to obtain $\left|C_{8}\right| \leq C \tau$. Finally, we extract the desired norms on the left hand side of (4.2) as follows

$$
\begin{gathered}
\int_{0}^{\sigma} \int_{\Omega} \epsilon\left(\partial_{t} \mathbf{E}_{N, h}(t)-\partial_{t} \mathbf{E}(t)\right) \cdot\left(\overline{\mathbf{E}}_{N, h}(t)-\mathbf{E}(t)\right) d x d t=\frac{1}{2}\left\|\mathbf{E}_{N, h}(\sigma)-\mathbf{E}(\sigma)\right\|_{\mathbf{L}_{\epsilon}^{2}(\Omega)}^{2} \\
-\frac{1}{2}\left\|\mathbf{E}_{0 h}-\mathbf{E}_{0}\right\|_{\mathbf{L}_{\epsilon}^{2}(\Omega)}^{2}+\int_{0}^{\sigma} \int_{\Omega} \epsilon\left(\partial_{t} \mathbf{E}_{N, h}(t)-\partial_{t} \mathbf{E}(t)\right) \cdot\left(\overline{\mathbf{E}}_{N, h}(t)-\mathbf{E}_{N, h}(t)\right) d x d t
\end{gathered}
$$

and

$$
\begin{aligned}
& \int_{0}^{\sigma} \int_{\Omega} \mu^{-1}\left(\partial_{t} \mathbf{B}_{N, h}(t)-\partial_{t} \mathbf{B}(t)\right) \cdot\left(\overline{\mathbf{B}}_{N, h}(t)-\mathbf{B}(t)\right) d x d t \\
& =\frac{1}{2}\left\|\mathbf{B}_{N, h}(\sigma)-\mathbf{B}(\sigma)\right\|_{\mathbf{L}_{1 / \mu}^{2}(\Omega)}^{2}-\frac{1}{2}\left\|\mathbf{B}_{0 h}-\mathbf{B}_{0}\right\|_{\mathbf{L}_{1 / \mu}^{2}(\Omega)}^{2} \\
& \quad+\int_{0}^{\sigma} \int_{\Omega} \mu^{-1}\left(\partial_{t} \mathbf{B}_{N, h}(t)-\partial_{t} \mathbf{B}(t)\right)\left(\overline{\mathbf{B}}_{N, h}(t)-\mathbf{B}_{N, h}(t)\right) d x d t .
\end{aligned}
$$

In view of (3.26), we have

$$
\begin{aligned}
& \left|\int_{0}^{\sigma} \int_{\Omega} \epsilon\left(\partial_{t} \mathbf{E}_{N, h}(t)-\partial_{t} \mathbf{E}(t)\right) \cdot\left(\overline{\mathbf{E}}_{N, h}(t)-\mathbf{E}_{N, h}(t)\right) d x d t\right| \\
\leq & \left\|\partial_{t} \mathbf{E}_{N, h}-\partial_{t} \mathbf{E}\right\|_{L^{1}\left((0, T), \mathbf{L}_{\epsilon}^{2}(\Omega)\right)}\left\|\overline{\mathbf{E}}_{N, h}-\mathbf{E}_{N, h}\right\|_{L^{\infty}\left((0, T), \mathbf{L}_{\epsilon}^{2}(\Omega)\right)} \\
\leq & C \tau\left(\left\|\partial_{t} \mathbf{E}\right\|_{L^{1}\left((0, T), \mathbf{L}_{\epsilon}^{2}(\Omega)\right)}+1\right) .
\end{aligned}
$$

By combining (4.10) and its analogue for $\mathbf{B}$ with the previously proved estimation for $C_{i}, i \in\{1, \ldots, 8\}$, and Lemma 4.3, we finally arrive at the desired error estimate. 
5. Numerical Results. We close this paper by presenting numerical results for some particular examples for (VI). When it comes to computing the solution (E, B) to (VI), Euler's implicit method provides an iterative algorithm, which also enables us to split the mixed problem into two associated problems as we did in Theorem 3.3. We recall (3.8), which gives an explicit formula for $\mathbf{B}_{h}^{n}$ :

$$
\mathbf{B}_{h}^{n}=\mathbf{B}_{h}^{n-1}-\tau \operatorname{curl} \mathbf{E}_{h}^{n} \text { a.e. in } \Omega,
$$

provided $\mathbf{E}_{h}^{n}$ is already computed. In view of (3.10), $\mathbf{E}_{h}^{n}$ solves an elliptic curl-curl variational inequality of the form

$$
a\left(\mathbf{E}_{h}^{n}, \mathbf{v}_{h}-\mathbf{E}_{h}^{n}\right)+\varphi^{n}\left(\mathbf{v}_{h}\right)-\varphi^{n}\left(\mathbf{E}_{h}^{n}\right) \geq\left\langle\tilde{\mathbf{f}}^{n}, \mathbf{v}_{h}-\mathbf{E}_{h}^{n}\right\rangle \quad \forall \mathbf{v} \in \mathbf{V}_{h} .
$$

We solve the above variational inequality using the semi-smooth Newton method (cf. [12]).

Our computational domain is the cube $\Omega=(-1,1)^{3}$ and we apply a circular current $\mathbf{f}: \mathbb{R}^{3} \rightarrow \mathbb{R}^{3}$ defined by

$$
\mathbf{f}(x, y, z)= \begin{cases}1 / R\left(0,-z /\left(y^{2}+z^{2}\right)^{1 / 2}, y /\left(y^{2}+z^{2}\right)^{1 / 2}\right) & \text { for }(x, y, z) \in \Omega_{p} \\ 0 & \text { for }(x, y, z) \notin \Omega_{p}\end{cases}
$$

to a cylindrical pipe coil $\Omega_{p}$ with height $h=1$ and inner radius $r=0,3$. The constant

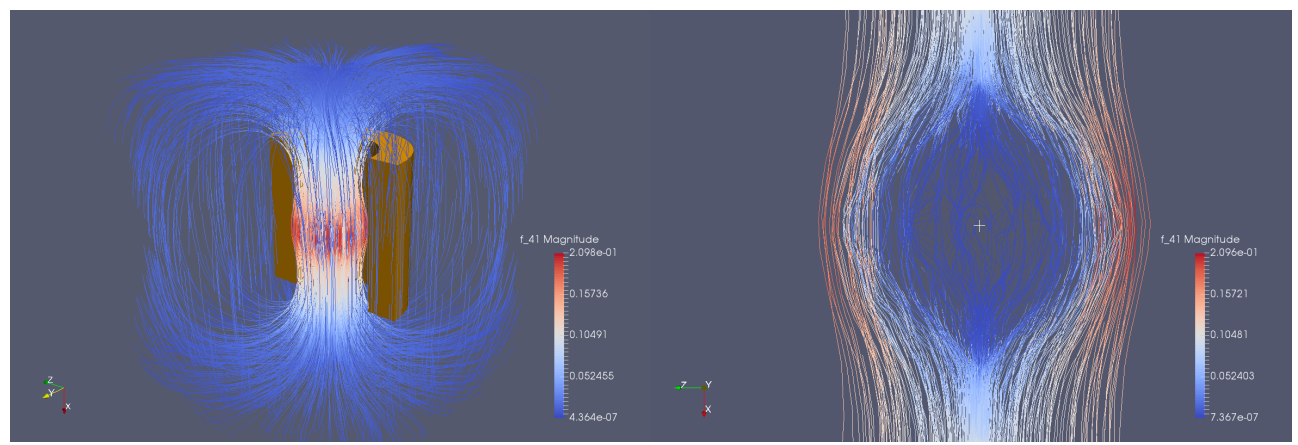

FIG. 1. First numerical example

$R>0$ denotes the electrical resistance of the pipe (here: $R=1$ ). All implementations were done with the open-source finite-element computational platform FENICS [19] and as a visualization tool PARAVIEw was used. For this study, the uniform tetrahedral mesh was refined around the coil. If we do not include a superconductor in this setup, the applied current induces an orthogonal magnetic field, which admits its greatest field strength inside the coil.

In the first example, we place a type-II superconducting ball $\Omega_{s c}$ with radius 0,2 in the center of the pipe and set $j_{c}=80 \chi_{\Omega_{s c}}, \epsilon=\mu=1$ as well as $\tau=1$ and $\left(\mathbf{E}_{0 h}, \mathbf{B}_{0 h}\right)=(0,0)$. This corresponds to the complementary system (A5) for the discrete inital electromagnetic fields. Once again, the mesh gets refined around the superconductor such that we end up with roughly 240.000 cells and 1.020 .000 degrees of freedom (DOFs) for the mixed finite element space. The resulting solution $\left(\mathbf{E}_{h}^{1}, \mathbf{B}_{h}^{1}\right)$ of (5.1)-(5.2) exhibits the physical phenomenon of the Meissner-Ochsenfeld effect. In 


\begin{tabular}{|c||c|c|c|c|c|c|c|}
\hline$n$ & 0 & 1 & 2 & 3 & 4 & 5 & 6 \\
\hline \hline$j_{c}\left(\cdot, \theta\left(t_{n}\right)\right)$ & $80 \chi_{\Omega_{s c}}$ & $50 \chi_{\Omega_{s c}}$ & $35 \chi_{\Omega_{s c}}$ & $20 \chi_{\Omega_{s c}}$ & $10 \chi_{\Omega_{s c}}$ & $5 \chi_{\Omega_{s c}}$ & $0,5 \chi_{\Omega_{s c}}$ \\
\hline$\theta\left(t_{n}\right)$ & $60,0 K$ & $65,0 K$ & $67,5 K$ & $70,0 K$ & $72,5 K$ & $75,0 K$ & $80,0 K$ \\
\hline$t_{n}$ & 0 & $1 / 6$ & $1 / 3$ & $1 / 2$ & $2 / 3$ & $5 / 6$ & 1 \\
\hline
\end{tabular}

Critical current $j_{c}$ and temperature $\theta$ of the superconductor at each the time step.

Figure 1, we see how the magnetic field lines get repelled by the superconductor and since they are squashed between the superconductor and the coil, one observes the highest magnetic field strength in this area (see Figure 1).

Keeping the observations of the first example in mind, we continue and compute a time-dependent problem, where the solution of the first example serves as the initial electromagnetic field, since it satisfies the complementary system (A5). We consider the temperature dependence in the critical current density $j_{c}$ for a superconductor with the nomial composition $\mathrm{Y}_{1.2} \mathrm{Ba}_{0.8} \mathrm{Cu}_{2} \mathrm{O}_{x}$ as it was suggested in [1]. Moreover, we set $\epsilon=\mu=1, T=1$ as well as $\tau=1 / 6$ and use the same amount of DOFs and cells as in the first example. We place the cooled down superconductor inside the coil in the same way it was done in the first example, but now the temperature increases over time (see Table 1), whereas the applied current source $\mathbf{f}$ stays constant. The evolution of the magnetic field over time is shown in Figure 2. One observes that the magnetic field lines in the squashed area start penetrating the superconductor as the temperature becomes larger and larger. As soon as the temperature $\theta$ exceeds the threshold $75 K$, the magnetical field completely penetrates the superconductor and we can no longer observe the Meissner-Ochsenfeld effect.

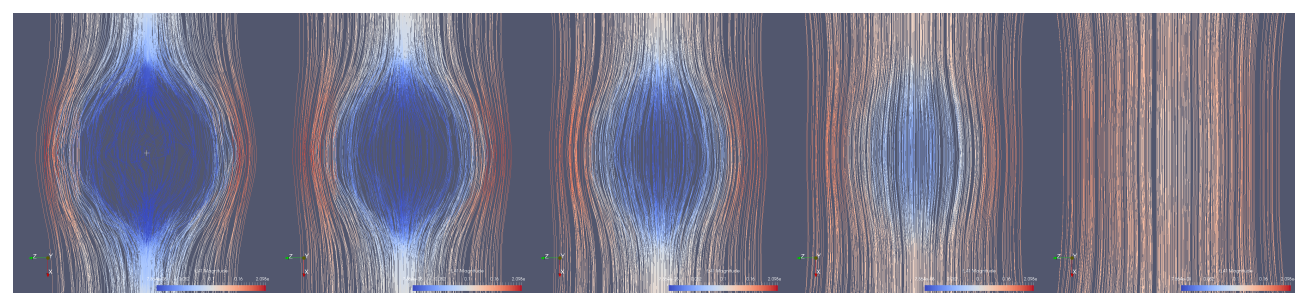

FIG. 2. Evolution of the magnetic field around the superconductor in the time-steps $t_{n}$ for $n \in\{0,1,2,3,4\}$.

Further Research. As pointed out in the introduction, the Bean critical-state model is a free boundary problem, as it involves unknown superconductive and normal regions, which may change their locations in course of time, depending on the temperature distribution $\theta$ and the applied current source $\mathbf{f}$. Thus, an adaptive mesh refinement strategy based on rigorous a posteriori error estimators will be useful, not only for increasing numerical accuracy, but also for capturing the unknown interfaces between the superconductive and normal regions. We also point out that Corollary 3.9 opens a way to study the temperature control in the magnetization process of type-II superconductivity. This leads to a state-constrained optimal control problem governed by a fully coupled system consisting of (VI) and non-smooth heat equations. This problem requires a substantial extension of the recently developed optimal control techniques for electromagnetic problems [25, 27]. 
[1] J. Aponte, H.C. Abache, A. Sa-Neto, and M. Octavio. Temperature dependence of the critical current in high- $\mathrm{T}_{\mathrm{c}}$ superconductors. Phys. Rev. B: Condens. Matter; (United States), 39:4, 21989.

[2] J. W. Barrett and L. Prigozhin. A quasi-variational inequality problem in superconductivity. Math. Models Methods Appl. Sci., 20(5):679-706, 2010.

3] J. W. Barrett and L. Prigozhin. Sandpiles and superconductors: nonconforming linear finite element approximations for mixed formulations of quasi-variational inequalities. IMA J. Numer. Anal., 35(1):1-38, 2015.

[4] C. P. Bean. Magnetization of hard superconductors. Phys. Rev. Lett., 8:250-253, Mar 1962.

[5] C. P. Bean. Magnetization of high-field superconductors. Rev. Mod. Phys., 36:31-39, Jan 1964.

[6] P. Ciarlet, Jr., H. Wu, and J. Zou. Edge element methods for Maxwell's equations with strong convergence for Gauss' laws. SIAM J. Numer. Anal., 52(2):779-807, 2014.

[7] P. Ciarlet, Jr. and J. Zou. Fully discrete finite element approaches for time-dependent Maxwell's equations. Numer. Math., 82(2):193-219, 1999.

[8] G. Deutscher and K. A. Müller. Origin of superconductive glassy state and extrinsic critical currents in high-T $\mathrm{c}_{\mathrm{c}}$ oxides. Phys. Rev. Lett.; (United States), 59:15, 1987.

[9] C. M. Elliott and Y. Kashima. A finite-element analysis of critical-state models for type-II superconductivity in 3D. IMA J. Numer. Anal., 27(2):293-331, 2007.

[10] C. M. Elliott, D. Kay, and V. Styles. A finite element approximation of a variational inequality formulation of Bean's model for superconductivity. SIAM J. Numer. Anal., 42(3):1324$1341,2004$.

[11] C. M. Elliott, D. Kay, and V. Styles. Finite element analysis of a current density-electric field formulation of Bean's model for superconductivity. IMA J. Numer. Anal., 25(1):182-204, 2005.

[12] K. Ito and K. Kunisch. Lagrange Multiplier Approach to Variational Problems and Applications. Society for Industrial and Applied Mathematics, 2008.

[13] F. Jochmann. On a first-order hyperbolic system including Bean's model for superconductors with displacement current. Journal of Differential Equations, 246(6):2151 - 2191, 2009.

[14] F. Kikuchi. On a discrete compactness property for the Nédélec finite elements. J. Fac. Sci. Univ. Tokyo Sect. IA Math., 36(3):479-490, 1989.

[15] S. Lang. Real and Functional Analysis. Springer-Verlag New York, 3 edition, 1993.

[16] J. Li. Error analysis of fully discrete mixed finite element schemes for 3-D Maxwell's equations in dispersive media. Comput. Methods Appl. Mech. Engrg., 196(33-34):3081-3094, 2007.

[17] J. Li and Y. Huang. Time-domain finite element methods for Maxwell's equations in metamaterials, volume 43 of Springer Series in Computational Mathematics. Springer, Heidelberg, 2013.

[18] J. L. Lions and G. Stampacchia. Variational inequalities. Communications on Pure and Applied Mathematics, 20(3):493-519, 1967.

[19] A. Logg, K.-A. Mardal, G. N. Wells, et al. Automated Solution of Differential Equations by the Finite Element Method. Springer, 2012.

[20] Ch. G. Makridakis and P. Monk. Time-discrete finite element schemes for Maxwell's equations. RAIRO Modél. Math. Anal. Numér., 29(2):171-197, 1995.

[21] P. Monk. Finite Element Methods for Maxwell's Equations. Numerical Analysis and Scientic Computation. Clarendon Press, 2003.

[22] J.-C. Nédélec. Mixed finite elements in $\mathbf{R}^{3}$. Numer. Math., 35(3):315-341, 1980.

[23] L. Prigozhin. On the Bean critical-state model in superconductivity. European Journal of Applied Mathematics, 7(3):237247, 1996.

[24] L. Prigozhin. Solution of thin film magnetization problems in type-II superconductivity. Journal of Computational Physics, 144(1):180 - 193, 1998.

[25] I. Yousept. Optimal Control of Quasilinear $\boldsymbol{H}$ (curl)-Elliptic Partial Differential Equations in Magnetostatic Field Problems. SIAM J. Control Optim., 51(5):3624-3651, 2013.

[26] I. Yousept. Hyperbolic Maxwell variational inequalities for Bean's critical-state model in type-II superconductivity. SIAM J. Numer. Anal., 55(5):2444-2464, 2017.

[27] I. Yousept. Optimal control of non-smooth hyperbolic evolution Maxwell equations in type-II superconductivity. SIAM J. Control Optim., 55(4):2305-2332, 2017.

[28] I. Yousept and J. Zou. Edge element method for optimal control of stationary Maxwell system with Gauss law. SIAM J. Numer. Anal., 55(6):2787-2810, 2017. 